\title{
Stromal Interaction Molecule 1 (STIM1) Knock- down Attenuates Migration and Proliferation and Enhances the Expression of Thyroid Specific Proteins in Human Follicular Thyroid Cancer Cells.
}

\section{Muhammad Yasir Asghar ( $\sim$ yasir.asghar@helsinki.fi)}

Minerva Foundation Institute for Medical Research, Biomedicum, Helsinki https://orcid.org/0000-00021935-8462

Taru Lassila

Åbo Akademi University; Minerva Foundation Institute

Ilkka Paatero

Turku Bioscience Centre, University of Turku and Åbo Akademi University

\section{Van Dien Nguyen}

Cardiff University School of Medicine

\section{Pauliina Kronqvist}

Department of Pathology, University of Turku, Finland

Jixi Zhang

Chongqing University College of Bioengineering

Anna Slita

Åbo Akademi University, Turku

Christoffer Löf

University of Turku: Turun Yliopisto

\section{You Zhou}

Cardiff University School of Medicine

Jessica Rosenholm

Åbo Akademi University, Turku

Kid Törnquist

Åbo Akademi University; Minerva Foundation Institute for Medical Research

\section{Original Article}

Keywords: Thyroid cancer, Stromal interaction molecule 1 (STIM1), ORAl1 calcium channel, Storeoperated calcium entry (SOCE), Migration and Invasion, Proliferation 
DOl: https://doi.org/10.21203/rs.3.rs-183367/v1

License: (c) (1) This work is licensed under a Creative Commons Attribution 4.0 International License. Read Full License 


\section{Abstract}

Stromal interaction molecule 1 (STIM1) and the ORAl1 calcium channel mediate store-operated calcium entry (SOCE) and regulate a multitude of cellular functions. The identity and function of these proteins in thyroid cancer remained elusive. We show that STIM1 and ORAI1 expression is elevated in thyroid cancer cell lines, compared with primary thyroid cells. Knock-down of STIM1 or ORAI1 attenuated SOCE, reduced migration, and expression of promigratory sphingosine 1-phosphate (S1P) and vascular endothelial growth factor-2 (VEGFR2) receptors in thyroid cancer ML-1 cells. Cell proliferation was attenuated in these knock-down cells due to increased G1 phase of the cell cycle and enhanced expression of cyclindependent kinase inhibitory proteins p21 and p27. STIM1 protein was upregulated in thyroid cancer tissue, compared with normal tissue. Downregulation of STIM1 restored expression of thyroid stimulating hormone receptor (TSHR), thyroid specific proteins and increased iodine uptake. STIM1 knockdown ML-1 cells were more susceptible to chemotherapeutic drugs, and significantly reduced tumor growth in Zebrafish. Furthermore, STIM1-siRNA-loaded mesoporous polydopamine nanoparticles attenuated migration and proliferation of ML-1 cells. Taken together, our data suggest that STIM1 is a potential diagnostic and therapeutic target for treatment of thyroid cancer.

\section{Introduction}

Thyroid cancer is the most prevalent endocrine cancer, the incidence and related mortality of which are increasing globally [1]. $95 \%$ of thyroid cancer forms arise from follicular cells [2, 3]. Usually, the prognosis of thyroid tumors is good. However, due to dedifferentiation or genetic mutations, highly aggressive phenotype arises, which potentiates metastasis of tumor cells mainly to lungs, brain and bones causing death of the patient within 6 months $[4,5]$. There is no rational treatment available for such tumors [6]. Thus, novel approaches to curtail and halt the progression of thyroid cancer to a metastatic and terminally fatal disease are urgently warranted.

Store-operated calcium entry (SOCE) is activated as a result of agonist-evoked emptying of calcium stores in the endoplasmic reticulum (ER). This mechanism results in activation of plasma membrane calcium channels and calcium entry into the cells, ensuring that the calcium stores will be replenished [7]. The calcium signals generated by the SOCE regulate a multitude of cellular functions, including cancer cell proliferation and migration [8-11].

Stromal interaction molecule 1 (STIM1) is localized in the ER and plasma membrane. It functions as an ER-calcium sensor protein and gets activated by ER calcium-depletion, oligomerizes with other STIM1 molecules, and recruits plasma membrane ORAl1 channels. This results in calcium entry into the cells and refilling of the ER-calcium stores [12]. STIM1 has been shown to activate also other channels, e.g. transient receptor potential canonical (TRPC) isoforms, including TRPC1 [13]. Previously, we have reported that TRPC1 functions in thyroid cancer cells as a receptor-operated calcium channel and modulates thyroid cancer cell migration and invasion [14]. 
Previous studies have indicated that there are relatively high expression levels of STIM and ORAI isoforms in several cancer cell types [15]. The expression and function of STIM1 and ORAI1 in thyroid cancer progression and migration have remained elusive.

In the present study, we investigated the expression of STIM1 and ORAI1 proteins in thyroid cells and their importance in regulating invasion and migration. We report that the expression of STIM1 is upregulated in all thyroid cancer cell lines studied compared to primary thyroid cells. Notably, STIM1 was upregulated in all thyroid cancer patient tissue samples investigated, as compared with normal thyroid tissue. In thyroid follicular ML-1 cancer cells, SOCE was significantly decreased in both stable STIM1 (STIM1-KD) and ORAI1 (ORAI1-KD) knock-down cells, compared with control mock-transfected cells. Furthermore, the basal invasion and migration were decreased, and the sphingosine 1-phosphate (S1P)-evoked invasion and migration was abolished in ML-1 cells after STIM1-KD or ORAI1-KD. In STIM1-KD cells, proliferation was reduced and the expression of $\mathrm{S}_{1} \mathrm{P}_{3}$, VEGFR2, and H1F-1 a was decreased, as well as the activity of matrix metalloproteinase - 2 and - 9 (MMP2 and MMP9). In a zebrafish xenograft model, STIM1-KD decreased tumor growth. Furthermore, we show that STIM1-KD increases the susceptibility of thyroid cancer ML-1 cells to drugs used for treatment of thyroid cancer, reinstates the expression of thyroid stimulating hormone receptor (TSHR), thyroid specific proteins and increased iodine uptake. Finally, use of modified mesoporous polydopamine (MPDA) nanoparticles was found to be a useful system for functional delivery of siRNA in thyroid cancer cells.

\section{Materials And Methods}

H6621 cell culture medium, supplements kit and gelatin-based coating solution were purchased from Cell Biologics (Chicago, IL, USA). DMEM, sodium pyruvate solution, BSA, fatty acid-free BSA, poly-l-lysine, puromycin, HEPES solution and JumpStart Taq DNA polymerase were purchased from Sigma-Aldrich (St.Louis, MO, USA). RPMI 1640 medium (without I-glutamine) was from Lonza (Basel, Switzerland). FBS, trypsin-EDTA solution, I-glutamine, penicillin/streptomycin (P/S), OptiMEM, and F-12 (Ham's nutrient medium) $\mathrm{GIBCO}^{\mathrm{TM}}$, RevertAid reverse transcriptase, RiboLock RNase inhibitor, random hexamer primers, dNTPs, GeneRuler 100-bp Plus DNA Ladder, HEPES, the bicinchoninic acid protein assay reagent kit Pierce $^{T M}$ and Alexa Fluor ${ }^{T M}$ Red Fluorescent Control siRNA were from Thermo Fisher Scientific (Waltham, MA, USA). Sphingosine 1-phosphate (S1P) was from Biomol (Plymouth, PA). Primary antibodies against $\mathrm{S}_{1} \mathrm{P}_{1}, \mathrm{~S}_{\mathrm{P}}, \mathrm{TG}, \mathrm{NIS}, \mathrm{TPO}$, and Hsc70 were from Santa Cruz Biotechnology, Inc. (Santa Cruz, CA). HRPconjugated goat anti-rabbit IgG and the Aurum total RNA isolation kit were from Bio-Rad (Hercules, CA, USA). Primary antibodies against VEGFR2, HIF-1a, p21waf1/cip1, p27kip1, cdk6, MMP2, ERK1/2, pERK1/2, E-cad, N-Cad, TSHR, and HRP-conjugated anti-rat and anti-mouse IgG were from Cell Signaling Technology (Denver, MA, USA). Primary antibody against STIM1, ORAI1, and calpain activity and MTT proliferation assay kits were purchased from Abcam (Cambridge, MA). Cell culture plastic ware and human collagen type IV were from Becton Dickinson (Franklin Lakes, NJ, USA), and Transwell inserts for migration assays were from Corning, Inc. (Corning, NY). All the chemicals and reagents used were of molecular biology and reagent grades. Fura-2 AM was from Molecular Probes (Eugene, OR). Thapsigargin 
was from Alexis Corporation (San Diego, CA). KAPA Probe Fast Master Mix was from Kapa Biosystems (Boston, MA), and the Universal Probe Library probes were from Roche (Basel Switzerland).

Cell Culture - Human primary thyroid epithelial cells were purchased from Cell Biologics (Chicago, IL, USA). The cells were cultured in complete human epithelial cell medium with supplements kit (H6621, cell biologics, Chicago, IL, USA). The cells were plated on gelatin-coated plates as per manufacturer's instructions. The human ML-1 follicular thyroid cancer cells were cultured in DMEM with $10 \%$ FBS, $1 \%$ penicillin/streptomycin (P/S), and 1\% L-glutamine. FTC-133 thyroid follicular cancer cells were purchased from Banca Biologica e Cell Factory (Genova, Italy). The cells were cultured in DMEM and F-12 (Ham's) medium (1:1) with 10\% FBS and 1\% L-glutamine and 1\% P/S. The C643 anaplastic thyroid cancer cells were provided by Dr Nils-Erik Heldin (Karolinska Institute, Stockholm, Sweden) and cultured in DMEM with $10 \%$ FBS, 1\% L-glutamine, and 1\% penicillin/streptomycin. The anaplastic thyroid cancer THJ-16T cells were acquired from Dr John Copland (Mayo Clinic, FL, USA). The cells were cultured in RPMI 1640 with $10 \%$ FBS, $1 \%$ penicillin/streptomycin, 1 mM sodium pyruvate, and $25.03 \mathrm{mM}$ HEPES. The cell cultures were maintained in a water saturated atmosphere supplemented by $5 \% \mathrm{CO} 2$ and $95 \%$ air at $37 \mathrm{C}^{\circ}$ in the incubators.

Generation of stable cell lines - Cells were grown on 12-well plates. The transduction was performed according to the manufacturer's instructions using non-targeting shRNA lentivirus particles, and STIM1or ORAl1-targeting lentiviral particles (Sigma, St. Louis, MO, USA). The sequences are provided in Supplementary Table.1. After 48 hours, the medium was changed to the medium containing $0.5 \mu \mathrm{g} / \mathrm{ml}$ Puromycin. The cells were cultured with puromycin containing medium hereafter. The knock-down of STIM1 and ORAI1 was measured on mRNA level by quantitative-PCR and on protein level by western blotting, respectively.

Transient transfections -4 million cells were pelleted and re-suspended in $400 \mu \mathrm{l}$ OptiMEM together with $20 \mu \mathrm{g}$ of the control siRNA, siSTIM1, pYFP, pSTIM1 or pORAl1 plasmids. The cells were electroporated at $975 \mu \mathrm{F}$ and $240 \mathrm{~V}$ and were grown in respective media for 48 hours before the start of the experiments.

Qualitative end-point PCR and quantitative realtime PCR - RNA was extracted with Aurum ${ }^{\mathrm{TM}}$ Total RNA Mini Kit (Bio-Rad; CA, USA) or by TRI reagent (Sigma Aldrich; St. Louis, MO, USA) according to the manufacturer's instructions. RNA integrity was checked by gel electrophoresis and RNA concentration and purity was determined with Nanodrop 2000 (Thermo Fisher Scientific; Waltham, MA) and NanoVue Plus (Healthcare Bio-Sciences AB; Uppsala, SE). cDNA samples were prepared with RevertAid reverse transcriptase and SuperScript IV $®$ Reverse Transcriptase (Thermo Fisher Scientific, MA, USA) from equal amounts of RNA. Reaction mixtures lacking either reverse transcriptase or RNA were used as negative controls. For primers information see supplementary Table. 1. Quantitative real-time PCR assays were designed with the Universal Probe Library (UPL) Assay Design Center (www.rocheappliedscience.com). PBGD was used as a reference gene. RT-PCR was performed with KAPA Probe Fast Master Mix and the StepOnePlus Real-Time PCR System (Applied Biosystems ${ }^{\text {TM }}$, Thermo fisher scientific, MA, USA) using the relative standard curve method. 
Western Blotting - The whole cell lysates and for the Western blotting were performed as described elsewhere [16]. In some experiments, equal amounts of proteins were loaded on 10\% Fast Cast Stain-free sodium dodecyl sulfate polyacrylamide gel electrophoresis (SDS-PAGE) gels. Blotting was performed using PVDF membranes on BioRad Transblot system. After blocking, the blots were probed overnight with primary antibodies. Proteins were detected with enhanced chemiluminescence (ECL; Thermo Scientific, 242 Waltham, MA). Densitometric analysis was performed using the ImageJ program for image analysis (National Institutes of Health, Bethesda, MD), and the results were corrected for protein loading by normalization with Hsc70 expression.

Measurements of $\left[\mathrm{Ca}^{2+}\right]_{i}$ in Single Cells - Cells were processed and analyzed as described elsewhere [17]. A HAMAMATSU digital camera C10600 ORCA- $R^{2}$ with controller (Photonics K.K.) was used to capture fluorescence images at $1-3 \mathrm{~s}$ to avoid bleaching. The images were acquired and processed using the Axon Imaging Workbench 6 software (INDEC BioSystems, Santa Clara, CA). The intracellular free calcium concentrations were calculated as ratio $\mathrm{F}_{340} / 380$.

Invasion Assays - Invasion assays were performed as described elsewhere [18]. Cells were serum starved by growing in $0.2 \%$ FAF-BSA-containing serum-free medium (SFM) overnight before the start of the invasion assays. In these experiments, the cells were stimulated with S1P and allowed to migrate towards $10 \%$ lipid-stripped FBS (LS-FBS) for $16 \mathrm{~h}$. In other experiments, cells were allowed to invade towards $10 \%$ FBS-containing culture medium for $16 \mathrm{~h}$. The non-migrated cells were removed with a cotton swab. The migrated cells were fixed in $2 \%$ paraformaldehyde for 10 min and then stained with $0.1 \%$ crystal violet in $20 \%$ methanol for $5 \mathrm{~min}$. The membranes were washed with PBS and water and allowed to dry overnight. The cells were counted at 40X magnification in a straight line bisecting the membrane.

Zymography - ML-1 MOCK and STIM1-KD cells were grown on 35-mm plates up to $80 \%$ confluency. The medium was changed to SFM medium for overnight. Next day, the medium was changed to fresh SFM medium ( $1 \mathrm{ml}$ on each plate) and the cells were stimulated with or without S1P (100 nM) for $6 \mathrm{~h}$. After 6 $\mathrm{h}$, the media were collected. Equal volumes of medium were mixed with loading buffer (0.1 $\mathrm{m}$ Trisphosphate buffer, $\mathrm{pH} 6.8$, containing $20 \%$ glycerol, $6 \%$ SDS, and $0.04 \%$ bromphenol blue). The samples were electrophoresed with the $10 \%$ SDS gels containing gelatin $(2.65 \mathrm{mg} / \mathrm{ml})$. The gels were incubated in Zymo buffer (50 mm Tris- $\mathrm{HCl}$ containing 2.5\% Tween 80 and $0.02 \mathrm{NaN}_{3}, \mathrm{pH} 7.5$ ) for $30 \mathrm{~min}$. The gels were then incubated in Zymo buffer containing $1 \mu \mathrm{m} \mathrm{ZnCl} 2$ and $5 \mathrm{~mm} \mathrm{CaCl}$ for $30 \mathrm{~min}$. For gelatinolytic activity gels were incubated at $37^{\circ} \mathrm{C}$ for overnight in buffer containing $50 \mathrm{~mm}$ Tris- $\mathrm{HCl}, 5 \mathrm{~mm} \mathrm{CaCl}, 1 \mu \mathrm{m}$ $\mathrm{ZnCl}_{2}$, and $0.02 \% \mathrm{NaN}_{3}(\mathrm{pH}$ 7.5). The gelatin degradation was visualized under UV light, and after that, the gels were stained with Coomassie Blue R250 for 1-2 $\mathrm{h}$. The gelatinolytic activity was visualized as clear bands against blue background on stained gels. The clear bands after destaining for $30 \mathrm{~min}$, as shown in (Figure. 5E) were quantified by the program ImageJ. The data were normalized with the respective total protein concentrations of the respective cells in the culture plates.

Calpain activity assays -2 million cells were grown on 100-mm plates. The cells were serum-starved overnight and stimulated with or without S1P $(100 \mathrm{nM})$ for $6 \mathrm{~h}$. Next, the cells were detached and washed 
three times with PBS. Thereafter, calpain activity assays were performed according to the manufacturer's instructions (Abcam, Cambridge, MA). The samples were analyzed at an excitation of $400 \mathrm{~nm}$ and emission at $505 \mathrm{~nm}$ using a fluorescence analyzer. The results were normalized with the respective total protein concentrations of each culture plate and presented as \% calpain activity.

Proliferation Assays - The $\beta$ H] thymidine incorporation method was used to study the proliferation of the ML-1 MOCK, STIM1-KD and ORAI1-KD cells. 50,000 cells were seeded on 35-mm plates and allowed to grow for $24 \mathrm{~h}$. Four hours prior to the end of each experiment, $0.4 \mu \mathrm{Ci} / \mathrm{ml}\left[{ }^{3} \mathrm{H}\right]$ thymidine was added. The cells were washed three times with PBS, incubated for 10 min with $5 \%$ trichloric acetic acid, and then incubated for 10 min with $0.1 \mathrm{~m} \mathrm{NaOH}$. The samples were transferred into scintillation tubes and high sample load scintillation cocktail Optiphase Hisafe 3 was added. The radioactivity was measured using a Wallac 1414 liquid scintillation counter.

MTT proliferation assay method 10,000 ML-1 MOCK and STIM1-KD cells were grown on 96 well plate for overnight and treated with indicated concentration of respective drugs for $24 \mathrm{~h}$. Thereafter, the assays were performed according to the manufacturer's instructions. The absorbances were measured at $590 \mathrm{~nm}$ using a spectrophotometer.

FACS Analysis - 0.5 million ML-1 Mock, STIM1-KD and ORAI1-KD cells were grown overnight on 35-mm plates. The cells were detached with EDTA-trypsin solution and were centrifuged. The cell pellets were suspended in $500 \mu$ of propidium iodide solution $(0.05 \mathrm{mg} / \mathrm{ml}$ propidium iodide, $3.8 \mu \mathrm{M}$ sodium citrate, $0.1 \%$ Triton X-100 in PBS) and incubated for $15 \mathrm{~min}$ at room temperature. The samples were then processed by flow cytometry using FACSCalibur and CellQuest Pro software (BD Biosciences, San Jose, CA). ModFit LT 4.1 software was used to calculate the percentage of cells in each phase of cell cycle.

lodine uptake Assays - The uptake of iodide in ML-1 MOCK and STIM1-KD cells was determined by a nonradioactive, fast and highly reproducible spectrophotometric method based on the catalytic effect of iodide on reduction of yellow cerium (IV) to colorless cerium (III) in the presence of arsenious acid (Sandell-kolthoff reaction). The assay was performed exactly as described elsewhere [19]. In some experiments, the cells were pre-incubated with $1 \mathrm{mU} / \mathrm{ml}$ for overnight before the start of the assay.

Synthesis and modification of MPDA - The mesoporous polydopamine (MPDA) particles were prepared by a templated synthesis method as described elsewhere [20]. The surface of the obtained MPDA nanoparticles were modified by $\mathrm{N}, \mathrm{N}$-dimethylethylenediamine (DMEA) presenting a tertiary amine group for facilitating the siRNA loading [21]. $1 \mathrm{mg}$ of MPDA nanoparticles were suspended in $2 \mathrm{ml}$ of Tris buffer (pH.8.5). After the introduction of DMEA $(25.6 \mathrm{mg})$, the mixture was stirred for 1 hour at room temperature. The DMEA modified MPDA nanoparticles were isolated from the suspension by centrifugation.

siRNA loading of DMEA modified MPDA nanoparticles - $0.1 \mathrm{mg}$ siRNA STIM1, non-target control siRNA or control siRNA-Alexa Fluro 555 were mixed with 1mg DMEA modified MPDA in $1 \mathrm{ml}$ of 2-(N-morpholino) ethanesulfonic acid (MES) buffer (final concentration 10 mM, pH 5.0) through continuous vibration 
mixing for 40 min. The siRNA loaded DMEA modified MPDA nanoparticles were separated by centrifugation at $11000 \mathrm{rpm}$ for $15 \mathrm{~min}$ and stored at -20هC. 50,000 ML-1 cells were grown on polylysine coated glass bottom $35 \mathrm{~mm}$ plate for overnight. Next day, $5 \mu \mathrm{M}$ control siRNA loaded or siRNA STIM1 loaded nanoparticles were added to respective culture plates. The cells were grown for $96 \mathrm{~h}$ to $120 \mathrm{~h}$. The cells were washed, and the coverslips were mounted with DAPI containing mounting media ProLong ${ }^{\text {TM }}$ Gold Antifade mountant with DAPI. The laser scanning confocal microscopy was performed using a Leica TCS SP8 X microscope (Leica Microsystems GmbH, Wetzlar, Germany) with 63x oil immersion magnification and a HC PL APO CS2 objective.

Zebrafish embryo xenograft - The procedure to carry-out xenotransplantation in zebrafish embryos was modified from as described elsewhere [22, 23]. Adult zebrafish (Danio rerio, casper strain (roy-/; mitfa-/-) [24] were maintained according to standard procedures [25]. The embryos were collected after natural spawning in breeding tanks. Experimentation with zebrafish was performed under license ESAVI/9339/04.10.07/2016. The zebrafish embryos were cultured in E3-medium ( $5 \mathrm{mM} \mathrm{NaCl}, 0.17 \mathrm{mM}$ $\mathrm{KCl}, 0.33 \mathrm{mM} \mathrm{CaCl}_{2}, 0.33 \mathrm{mM} \mathrm{MgSO}_{4}$ ) supplemented with $0.2 \mathrm{mM}$ phenylthiourea (PTU, Sigma-Aldrich) at $33^{\circ} \mathrm{C}$. Two days post-fertilization, the embryos were anesthesized with MS-222 (200 mg/l, SigmaAldrich) and mounted into low-melting point agarose for tumor transplantation.

Prior to transplantation, ML-1 cells were labelled with $5 \mu \mathrm{M}$ CellTracker Green CMFDA (Thermofisher Scientific), trypsinized and washed with PBS. Approximately 5-10 $\mathrm{nl}$ of ML-1 cell suspension was microinjected into yolk or pericardial cavity of the embryo using CellTramVario (Eppendorf), Injectman $\mathrm{Ni} 2$ (Eppendorf) micromanipulator and self-made borosilicate glass needles pulled from glass capillaries (TW100-4, World Precision Instruments Ltd., Sarasota, FL) using micropipette puller (PB-7, Narishige, Tokyo, Japan). After injections, the agarose gel was broken and embryos were released, washed with E3 and cultured in E3-PTU at $33^{\circ} \mathrm{C}$. On 1 day post-injection $(1 \mathrm{dpi})$, the successfully xenografed healthy embryos were selected to the experiment and placed into 12-well or 96-well plates (1 embryo per well). The embryos were anesthesized and imaged at $1 \mathrm{dpi}$ and $4 \mathrm{dpi}$ using Zeiss AxioZoom fluorescence stereomicroscope of Nikon Eclipse widefield microscope.

The size of the tumor was determined by manually measuring the fluorescent tumor area using FIJI software (ImageJ version $1.49 \mathrm{k}$ ) [26]. Samples having significant malformations, died embryos and images where embryo was not laterally oriented or out-of-focus were excluded from the analysis. Samples were not blinded.

STIM1 staining of patient samples - The use of tissue samples and related data was approved by Auria Biobank (decision AB19-2988) and the Hospital District of Southwest Finland and Turku University Hospital (decision T12/011/20). The samples were from both female and male patients ( 9 anaplastic thyroid carcinoma, 29 follicular thyroid carcinoma, and 138 papillary thyroid carcinoma). The age distribution of the patients was 18-88 years of age. Thyroid tissue was prepared according to standard histology practice, using buffered formalin ( $\mathrm{pH}$ 7.0) for fixation and embedding tissue into paraffin blocks. Tissue microarrays (TMAs) were prepared from tumour area of each patient. As controls, normal 
thyroid tissue samples (control) were obtained from 104 patients. Tissue sections were cut and stained with anti-STIM1 antibody (1/200; Abcam 57834) using Labvision Autostainer (Thermo-Fisher Scientific, Fremont, CA, USA) and detected with an Orion 2 step detection system using goat anti mouse/rabbit HRP + WellMed T100 HRP. Final images were acquired at 20X magnification using the case viewer software (HISTECH Ltd, Hungary).

High throughput data mining and Gene expression analyses - The clinical and transcriptomic data of the Thyroid Carcinoma (THCA) cohort were downloaded from the GDC database (https://portal.gdc.cancer.gov/projects/TCGA-THCA). We included 502 thyroid tumors and 58 adjacent normal samples for transcriptome analysis. Among those tumor samples, 353 were papillary thyroid tumors while 106 were follicular thyroid tumors. To assess gene expression levels in normal thyroid samples, we retrieved gene expression data (as raw count values) of 563 thyroid samples from the Genotype-Tissue Expression (GTEx) (https://gtexportal.org/home) for comparative analysis of gene expression. Only genes that express in at least $50 \%$ of the samples were assessed. DESeq2 was used for differential expression analysis between thyroid cancer groups and their matched para-cancerous samples [27]. A Benjamini-Hochberg adjusted $p$-value of $<0.05$ was considered as statistical significance. The expression profiles of genes of interest (STIM1 and ORAI1) were also extracted from both retrieved datasets after performing variance stabilizing (VST) transformation for comparative analyses between cancerous tissues, cancer adjacent normal tissues, and controls.

Statistical analysis - The results are presented as means \pm the standard error of the mean from at least three independent measurements. Student's $t$ test was used when two means were compared. One-way analysis of variance and Bonferroni's post hoc test were applied when three or more means were compared. Kruskal-Wallis test, Kolmogorov-Smirnov test and Fisher exact test were used to analyze nonnormally distributed data. GraphPad Prism 8 program (GraphPad Software Inc., San Diego, CA) was used for the statistical analyses. $p$ values $<0.05$ were considered statistically significant.

\section{Results}

\section{The expression of STIM1 and ORAl1 channels is increased in thyroid cancer cell lines}

Increasing evidence suggest that store-operated calcium entry (SOCE), mediated by the STIM1 and ORAI1 proteins, is a key player in regulating the pathophysiology of cancer $[28,29]$. However, the expression and function of these key signaling proteins in human thyroid cancer has remained elusive. We show here that the protein level of STIM1 is higher in human follicular and anaplastic cancer cell lines, and that ORAI1 is higher in follicular cancer cell lines, compared with normal human thyroid primary cells (Fig. 1A). Based on these results, we then investigated the importance of the STIM1 and ORAl1 channels in follicular thyroid cancer ML-1 cells, a cell line showing the highest expression of STIM1 and ORAI1 compared to normal human thyroid cells. We generated stable STIM1 and ORAl1 knock-down (STIM1-KD, ORAl1-KD) ML-1 cell lines (Fig. 1B-E) 


\section{Knock-down of STIM1 or ORAl1 attenuates store-operated calcium entry (SOCE)}

Previous studies have established that STIM1 forms a complex with ORAI1 channels to evoke SOCE [22, 25]. Thus, we first investigated this mechanism in ML-1 cells. As can be seen in (Fig. 2A-2C), a significant difference was observed in both the thapsigargin $(\mathrm{Tg})$-evoked calcium transient and on $\mathrm{Ca} 2+$ entry when $\mathrm{Ca} 2+$ was re-added to Tg-treated STIM1-KD cells, compared to the control MOCK cells. There was no significant difference in the Tg-evoked calcium transient in ORAl1-KD cells compared to MOCK cells as confirmed by calculating the area under the Tg-evoked calcium curve, as shown in (Fig. 2D-F). However, there was a significant decrease in $\mathrm{Ca} 2$ + entry when $\mathrm{Ca} 2$ + was re-added to the Tg-treated ORAl1-KD cells, compared to the MOCK cells (Fig. 2D, G). Thus, both STIM1 and ORAI1 are important in regulating SOCE in thyroid cancer cells.

\section{Knock-down of STIM1 or ORAl1 decrease migration and receptor expression in thyroid cancer ML-1 cells}

STIM1 and ORAI1 have been reported to regulate migration of cancer cells $[29,30]$. We have previously characterized sphingosine 1-phosphate (S1P) and VEGFR2 signaling in thyroid cancer cells. S1P activates migration of ML-1 cancer cells via promigratory receptors S1P1, S1P3 and VEGFR2 [16, 31]. Thus, we investigated the effects of STIM1 or ORAl1 knock-down on ML-1 cancer cell migration. As can be seen in (Fig. 3A, D), both basal and S1P-evoked migration was attenuated in both the STIM1-KD and ORAI1-KD cells, compared with MOCK cells. However, these effects of STIM1 or ORAl1 knock-down on basal and S1P-evoked migration were reversible (supplementary Fig. 1A and B). To explain this abolished effect of S1P on STIM1-KD and ORAI1-KD cells, we determined S1P and VEGFR2 receptor expression. The results showed that S1P3 and VEGFR2 were downregulated in STIM1-KD cells (Fig. 3B, C). In ORAl1KD cells, S1P1 and VEGFR2 were downregulated (Fig. 3E, F). Thus, both STIM1 and ORAl1 are important regulators of thyroid cancer cell migration.

\section{Knock-down of STIM1 and ORAl1 modulate ML-1 cell proliferation}

Evidence have shown that STIM1 and ORAl1 regulate the proliferation in different cancers, including brain, ovarian, pancreatic, nasopharyngeal, and renal cancers [32]. We next investigated the effect of STIM1 and ORAI1 knock-down on ML-1 cell proliferation. The results show that the proliferation was significantly attenuated in both STIM1 and ORAI1 knock-down cells, compared with MOCK cells (Fig. 4A). The effects of STIM1 or ORAI1 knock-down on proliferation were reversible (supplementary Fig. 1C). We next investigated the mechanism by which STIM1 or ORAl1 knock-down cells decreased proliferation. We first analyzed the cell cycle by FACS analysis. In both STIM1-KD and ORAI1-KD cells, there was a significant increase in cell population in the $\mathrm{G}_{1}$ phase and a significant decrease in the $S$ phase of the cell cycle, compared with the MOCK cells (Fig. 4B, C). We also investigated the expression of the cell cycle 
regulatory proteins $\mathrm{p} 21^{\mathrm{kip} 1}$ and $\mathrm{p} 27^{\mathrm{waf} 1 / \mathrm{cip} 1}$ and observed that these proteins were significantly increased in the knock-down cells (Fig. 4D, E). Furthermore, cdk6 was significantly decreased in both STIM1-KD and ORAI1-KD cells, compared with MOCK cells (Fig. 4E).

As we observed a decrease in proliferation in STIM1-KD cells, we further examined if the effect of the chemotherapeutic drugs Lenvatinib, paclitaxel and doxorubicin on ML-1 cell proliferation and on STIM1KD ML-1 cells. These drugs attenuated proliferation in a concentration-dependent manner, and the effect was enhanced in STIM1-KD cells (Fig. 4F-H). This is an important observation and suggests a novel approach to design the treatment of thyroid cancer with these drugs.

\section{STIM1 knock-down affects the migratory signaling pathway, EMT marker and thyroid specific proteins}

Previously, we have shown that HIF1a is downregulated in TRPC1 knock-down cells [14]. As can be seen in Fig. 5A, HIF1a expression was significantly downregulated in STIM1-KD cells. Next, we investigated the effects of STIM1-KD on epithelial mesenchymal transition (EMT) marker proteins. We found an upregulation of E-Cadherin protein and a decrease in vimentin and N-Cadherin in STIM1-KD cells compared to the MOCK ML-1 cells (Fig. 5B). In addition, the expression of total ERK1/2 and phosphorERK1/2 were downregulated (Fig. 5C). Recently, we have reported that S1P increased calpain activity and activity of MMP2 and MMP9 through $\mathrm{S}_{1} \mathrm{P}_{1,3}$. and that knock-down of TRPC1 decreased the secretion and activity of both MMP2 and MMP9 in ML-1 cells $[14,33]$. In STIM1-KD cells, the basal calpain activity and the activity of MMP2 and MMP9 were significantly decreased, and the S1P-evoked increase in calpain activity and activity of MMP2 and MMP9 were abolished (Fig. 5D, E). This, in part, explains the decreased migration of STIM1-KD cells.

Thyroid-specific genes including thyroid-stimulating hormone receptor (TSHR), sodium/iodide symporter (NIS), thyroperoxidase (TPO) and thyroglobulin (TG) are downregulated in thyroid cancer [34]. Therefore, we examined the expression of these proteins in STIM1-KD cells. Interestingly, these proteins were upregulated in STIM1-KD cells (Fig. 5F). In addition, both the basal and TSH-evoked (final concentration 1 $\mathrm{mU} / \mathrm{ml}$ ) iodine-uptake was increased in STIM1-KD cells (Fig. 5G).

\section{MPDA-DMEA nanoparticles, an efficient siRNA delivery system in ML-1 cells}

Nanoparticles have been shown to deliver siRNA or drugs into the thyroid cancer cells precisely and effectively $[35,36]$. Therefore, we used control siRNA Alexa 555 Fluor loaded MPDA-DMEA nanoparticles in ML-1 thyroid cancer cells. These nanoparticles were efficiently taken up by the cells and that siRNA was released after $96 \mathrm{~h}$ (Fig. 6A-C). In control experiments, we showed that the STIM1-siRNA works efficiently to downregulate STIM1 protein in ML-1 cells (Fig. 6D). We observed the same effect by using siRNA-STIM1 loaded MPDA-DMEA nanoparticles (Fig. 6E). Next, we investigated the effect of the empty, or siRNA loaded nanoparticles on ML-1 cell invasion and proliferation. There was no effect of empty 
nanoparticles, however, both the invasion and proliferation were significantly downregulated by STIM1siRNA loaded nanoparticles (Fig. 6F, G).

\section{Zebrafish embryos with human thyroid tumor cells- xenograft}

To analyze the effects of STIM1 knock-down on tumor growth in vivo, we transplanted MOCK and STIM1KD cells into the zebrafish embryos. The embryos were kept in 96-well plates (1 embryo/well) to allow longitudinal analysis of tumor growth. STIM1-KD ML-1 cells exhibited strongly reduced tumor growth, also confirmed by calculating the tumor area and fold change (Fig. 7A-D). Taken together, these data indicate STIM-1 is required for full tumorigenic potential of ML-1- cells.

\section{Analysis of STIM1 and ORAl1 gene and protein expression in human thyroid}

Expression of STIM1 in normal and thyroid cancer patient samples - A higher expression of STIM1 has been suggested to correlate with poor prognosis and survival in many cancers [37]. We investigated the expression of STIM1 in thyroid cancer patient samples and obtained 176 thyroid cancer patient tissue samples and 104 normal thyroid tissue samples (normal tissue of same thyroid cancer patient as well as normal thyroid tissue from healthy subjects) as described in the method section. The results showed an increased STIM1 staining in all types of thyroid cancer patient samples, compared to respective patient's normal thyroid tissue adjacent to the tumor, or with healthy subjects' normal thyroid tissue sections (Fig. 8A-G).

Differential gene expression in thyroid cancer versus normal thyroid tissues - STIM1 and ORAl1 gene expression was evaluated in thyroid tumor tissues $(n=502)$ versus normal thyroid solid tissues $(n=58)$ datasets in the TCGA Thyroid Carcinoma cohort. The expression of both STIM1 and ORAl1 genes was significantly upregulated in both the papillary and combined thyroid cancer tissues (Total) compared to tumor adjacent normal thyroid tissues (NT). However, we found STIM1 borderline upregulated $(p=0.058)$ but not ORAI1 in follicular thyroid cancer tissues (Fig. 8H). Furthermore, a comparison of STIM1 expression in normal thyroid solid tissues (GTEX dataset), thyroid tumor tissues (TP) and tumor adjacent normal thyroid tissues (NT) was determined. Interestingly, we observed that STIM1 was significantly upregulated not only in thyroid tumor tissues (TP) but also in the tumor adjacent thyroid tissues (NT) compared to the normal thyroid tissues (GTEX) (Fig. 8I). In another comparison, we found the STIM1 expression was significantly higher in follicular thyroid cancer tissues compared to the papillary thyroid cancer tissues (Fig. 8J).

\section{Discussion}

The hallmarks of cancer include uncontrolled growth, migration and invasion of the cancer cells $[38,39]$. For these processes calcium and calcium channels have been shown to play important roles [11, 40, 41]. 
We have previously shown that calcium entry is important for the migration and proliferation of follicular thyroid cancer ML-1 cells [14]. The STIM- and ORAI proteins have also been considered as novel targets for cancer therapy $[15,32]$. We show here that, especially STIM1, but to some extent also ORAI1, are upregulated in several thyroid cancers cell lines, compared with normal thyroid cells. This has also been shown in e.g. therapy resistant ovarian cancer cell lines [42]. Furthermore, STIM1 was upregulated in all studied patient samples of papillary, follicular, and anaplastic thyroid cancer, compared with healthy thyroid tissues from the same patients or from normal healthy subjects. Similar results have been obtained in several other cancers, including cervical cancer [43], melanoma [44], and colorectal cancer [45]. The differential STIM1 and ORAI1 gene expression analysis data clearly showed an upregulation of these genes in most of the thyroid cancer tissues compared to normal thyroid tissues datasets. Surprisingly, an increased STIM1 gene expression but not ORAI1 was observed in the tumor adjacent normal tissue compared to the normal thyroid tissue, indicating a possible role of STIM1 in the initiation of thyroid tumor formation. A higher STIM1 expression in follicular thyroid cancer tissues compared to the papillary thyroid cancer tissues datasets suggests that STIM1 is important in development of an aggressive thyroid cancer phenotype.

Downregulation of STIM1 or ORAI1 efficiently attenuated both proliferation and invasion of ML-1 cells, and the cell cycle regulators p21, p27, and Cdk6. In cervical cancer, inhibition of STIM1 resulted in increased expression of $\mathrm{p21}$, and arrested the cells in the $\mathrm{S}$ - and G2/M phase of the cell cycle [43]. In our experiments STIM1-KD and ORAI1-KD halted cells in the G1 and S phase. This was also observed in TRPC1-KD ML-1 cells [14]. We next investigated the effects of STIM1-KD in Zebrafish embryos [46, 47]. By injecting control ML-1 cells in the zebrafish xenograft experiment, the cells grew into a clear tumor; however, the STIM1-KD cells were unable to mount the growth of the tumor. This experiment shows that also in an in vivo setting, STIM1-KD cells were unable to proliferate and form a tumor.

In addition to basal migration, STIM1 and ORAl1 knock down cells reduced the S1P-evoked migration. As shown previously, S1P is an important enhancer of cancer cell migration [48], including thyroid cancer cells [16]. In both knock down cell lines, the expression of VEGFR2 was decreased, whereas SIP3 was decreased in STIM1-KD cells, and S1P1 in ORAI1-KD cells. These receptors are crucial for S1P-evoked migration [49]., In melanoma STIM1 blockade the recycling of MT1-MMP to the plasma membrane, thus inhibiting the breakdown of the extracellular matrix [44]. Furthermore, in STIM1-KD cells the S1Pstimulated extrusion of MMP2 and - 9 was attenuated, and HIF-1a, an important regulator of ML-1 cell migration [50], was reduced. The results are in line with our previous study, showing that in TRPC1-KD ML-1 cells, the expression of VEGFR2, S1P3, MMP2 and - 9, and HIF-1a were all decreased [14]. Collectively these results show that calcium fluxes play a vital role for the regulation of factors involved in the proliferation and migration in thyroid cancer cells.

In addition to surgery, pharmacological interventions are important in treating thyroid cancer [51]. We show that three commonly used drug, i.e., lenvatinib, paclitaxel and doxorubicin, efficiently attenuated proliferation of the ML-1 cells. Notably, the effect of these drugs was enhanced in STIM1-KD cells. A similar observation was made in STIM1-KD pancreatic adenocarcinoma cells [52]. These results suggest 
that a combination of chemotherapy and knock down or blocking of STIM could be effective in treating thyroid cancer. However, the use of pharmacological blockers or siRNA in the inhibition of e.g., STIM1 is problematic, considering the ubiquitous expression of this protein. To avoid this, nanoparticles carrying drugs or siRNA can be an option [53]. This has been proposed for thyroid cancer cells [35]' [54]. We have previously shown that drug-loaded nanoparticles can efficiently induce apoptosis of thyroid cancer cells [36]. Here we show that siRNA-loaded nanoparticles can be used successfully to knock down STIM1, and thereby attenuate both proliferation and migration of the ML-1 cells. A combination of siRNA-loaded nanoparticles and lower doses of e.g., doxorubicin could be a novel approach to curtail thyroid cancer progression, with less adverse effects of the chemotherapy.

An interesting observation was the fact that knocking down STIM1 increased the TSH receptor expression. In rat thyroid FRTL-5 cells, knock down of TRPC2 also induced an increase in TSH receptor expression [17]. Further, we observed an increased expression of the thyroid specific proteins thyroglobulin, thyroperoxidase, and the Na-I symporter in STIM1-KD cells. STIM1 knock down enhanced TSH-evoked uptake of iodine, decreased vimentin and $\mathrm{N}$-cadherin expression, and elevated E-cadherin expression. Previously, knock-down of STIM1 has been shown to suppress the expression of proteins important for epithelial-mesenchymal transition and enhance the expression of E-cadherin in human prostate cancer cells [55]. These results suggest that the ML-1 cells obtained a more normal thyroid phenotype, with re-differentiation of the cells [56]. The precise mechanisms by which STIM1-KD regulates the expression of these proteins remain to be established. Notably, as STIM1-KD increased TSH-evoked uptake of iodine, radioactive iodine could be used to destroy thyroid cancer cells.

\section{Conclusions}

We have shown here using different approaches and gene downregulation in thyroid cancer cells that calcium signaling and the STIM1 protein play an important function in driving thyroid cancer cell proliferation and migration, and that blocking calcium entry pathways by pharmacological means or using siRNA-loaded nanoparticles effectively inhibit proliferation and migration of the cells. In view of this, we propose that the combination of drugs and siRNA targeting could be a useful approach to design better treatments for thyroid cancer and for the benefit of patients afflicted by aggressive, metastatic forms of thyroid cancer.

\section{Declarations}

\section{Acknowledgements}

We want to thank Drs. Dan Lindholm and Vesa Olkkonen for constructive criticism of the manuscript. The study benefited from samples and data from the Auria Biobank, Turku, Finland. Merja Perälä (Auria Biobank) is thanked for help with the application paperwork. Eeva Jääskeläinen is thanked for expert technical assistance. Konrad Patyra is thanked for help with histology-slide scanner. 


\section{Authors' contributions}

M.Y.A. and K.T., conceived the research, supervised the work, and edited the manuscript, which was written by M.Y.A., and K.T. M.Y.A., T.L., I.P., V.D.N., Y.Z., and A.S carried out the experimental work and analyzed data. C.L. provided reagents and methodology. J.R. and J.Z. designed reagents and provided methodology. K.T., obtained the tissue specimens of human subjects. M.Y.A, P.K., and K.T. analyzed immunohistology data and M.Y.A., acquired final images. P.K analyzed and scored all normal and thyroid cancer patient samples. M.Y.A, T.L. and K.T obtained funding for this project. All authors read and approved the final submitted form of the manuscript.

\section{Funding}

This work was financially supported by the research grants awarded from Åbo Akademi University (M.Y.A., T.L.), Finnish-Norwegian Medical Foundation (M.Y.A), Sigrid Juselius Foundation (K.T.), Magnus Ehrnrooth Foundation (K.T.), and Liv och Hälsa Foundation (K.T).

\section{Availability of data and materials}

All the data and materials on which the conclusions of this paper are based have been included in the manuscript.

\section{Ethics approval and consent to the participate and publication}

The approval was obtained by Auria Biobank (decision AB19-2988) and the Hospital District of Southwest Finland and Turku University Hospital (decision T12/011/20).

\section{Declaration of Interests}

The authors declare that they have no known competing financial interests or personal relationships that could have appeared to influence the work reported in this paper.

\section{References}

1. Vigneri, R.; Malandrino, P.; Russo, M. Is Thyroid Cancer Increasing in Incidence and Aggressiveness? J. Clin. Endocrinol. Metab.2020, 105, e2639-e2640.

2. Dralle, H.; MacHens, A.; Basa, J.; Fatourechi, V.; Franceschi, S.; Hay, I.D.; Nikiforov, Y.E.; Pacini, F.; Pasieka, J.L.; Sherman, S.I. Follicular cell-derived thyroid cancer. Nat. Rev. Dis. Prim.2015, 1.

3. Elisei, R. Thyroid carcinoma. In Encyclopedia of Endocrine Diseases; 2018 ISBN 9780128122006.

4. Nagaiah, G.; Hossain, A.; Mooney, C.J.; Parmentier, J.; Remick, S.C. Anaplastic thyroid cancer: a review of epidemiology, pathogenesis, and treatment. J. Oncol.2011, 2011, 542358. 
5. Nikiforova, M.N.; Lynch, R.A.; Biddinger, P.W.; Alexander, E.K.; Dorn, I.G.W.; Tallini, G.; Kroll, T.G.; Nikiforov, Y.E. RAS point mutations and PAX8-PPAR(gamma) rearrangement in thyroid tumors: Evidence for distinct molecular pathways in thyroid follicular carcinoma. J. Clin. Endocrinol. Metab.2003, 88, 2318-2326.

6. Smallridge, R.C.; Marlow, L.A.; Copland, J.A. Anaplastic thyroid cancer: Molecular pathogenesis and emerging therapies. Endocr. Relat. Cancer 2009, 16, 17-44.

7. Putney, J.W. A model for receptor-regulated calcium entry. Cell Calcium1986.

8. Jardin, I.; Rosado, J.A. STIM and calcium channel complexes in cancer. Biochim. Biophys. Acta - Mol. Cell Res. 2016.

9. Mo, P.; Yang, S. The store-operated calcium channels in cancer metastasis: from cell migration, invasion to metastatic colonization. Front. Biosci. - Landmark2018.

10. Putney, J.W.; Steinckwich-Besançon, N.; Numaga-Tomita, T.; Davis, F.M.; Desai, P.N.; D’Agostin, D.M.; Wu, S.; Bird, G.S. The functions of store-operated calcium channels. Biochim. Biophys. Acta - Mol. Cell Res. 2017.

11. Fiorio Pla, A.; Kondratska, K.; Prevarskaya, N. STIM and ORAI proteins: Crucial roles in hallmarks of cancer. Am. J. Physiol. - Cell Physiol. 2016.

12. Roos, J.; DiGregorio, P.J.; Yeromin, A. V.; Ohlsen, K.; Lioudyno, M.; Zhang, S.; Safrina, O.; Kozak, J.A.; Wagner, S.L.; Cahalan, M.D.; et al. STIM1, an essential and conserved component of store-operated Ca 2+ channel function. J. Cell Biol.2005.

13. Yuan, J.P.; Zeng, W.; Huang, G.N.; Worley, P.F.; Muallem, S. STIM1 heteromultimerizes TRPC channels to determine their function as store-operated channels. Nat. Cell Biol.2007.

14. Asghar, M.Y.; Magnusson, M.; Kemppainen, K.; Sukumaran, P.; Löf, C.; Pulli, I.; Kalhori, V.; Törnquist, K. Transient receptor potential canonical 1 (TRPC1) channels as regulators of sphingolipid and VEGF receptor expression: Implications for thyroid cancer cell migration and proliferation. J. Biol. Chem.2015.

15. Chalmers, S.B.; Monteith, G.R. ORAI channels and cancer. Cell Calcium 2018.

16. Balthasar, S.; Samulin, J.; Ahlgren, H.; Bergelin, N.; Lundqvist, M.; Toescu, E.C.; Eggo, M.C.; Törnquist, K. Sphingosine 1-phosphate receptor expression profile and regulation of migration in human thyroid cancer cells. Biochem. J.2006, 398, 547-556.

17. Löf, C.; Sukumaran, P.; Viitanen, T.; Vainio, M.; Kemppainen, K.; Pulli, I.; Näsman, J.; Kukkonen, J.P.; Törnquist, $\mathrm{K}$. Communication between the calcium and cAMP pathways regulate the expression of the TSH Receptor: TRPC2 in the center of action. Mol. Endocrinol.2012.

18. Asghar, M.Y.; Viitanen, T.; Kemppainen, K.; Törnquist, K. Sphingosine 1-phosphate and human ether-a'go-go-related gene potassium channels modulate migration in human anaplastic thyroid cancer cells. Endocr. Relat. Cancer2012, 19, 667-680.

19. Waltz, F.; Pillette, L.; Ambroise, Y. A nonradioactive iodide uptake assay for sodium iodide symporter function. Anal. Biochem.2010. 
20. Chen, F.; Xing, Y.; Wang, Z.; Zheng, X.; Zhang, J.; Cai, K. Nanoscale polydopamine (PDA) meets $\pi-\pi$ Interactions: An interface-directed coassembly approach for mesoporous nanoparticles.

\section{Langmuir2016.}

21. Wang, Z.; Wang, L.; Prabhakar, N.; Xing, Y.; Rosenholm, J.M.; Zhang, J.; Cai, K. CaP coated mesoporous polydopamine nanoparticles with responsive membrane permeation ability for combined photothermal and siRNA therapy. Acta Biomater.2019.

22. Paatero, I.; Alve, S.; Gramolelli, S.; Ivaska, J.; Ojala, P. Zebrafish Embryo Xenograft and Metastasis Assay. BIO-PROTOCOL2018.

23. Pekkonen, P.; Alve, S.; Balistreri, G.; Gramolelli, S.; Tatti-Bugaeva, O.; Paatero, I.; Niiranen, O.; Tuohinto, K.; Perälä, N.; Taiwo, A.; et al. Lymphatic endothelium stimulates melanoma metastasis and invasion via MMP14-dependent Notch3 and $\beta 1$-integrin activation. Elife2018.

24. White, R.M.; Sessa, A.; Burke, C.; Bowman, T.; LeBlanc, J.; Ceol, C.; Bourque, C.; Dovey, M.; Goessling, W.; Burns, C.E.; et al. Transparent Adult Zebrafish as a Tool for In Vivo Transplantation Analysis. Cell Stem Cel/2008.

25. Nusslein-Volhard, C. and R.D. Zebrafish (Practical Approach Series). OUP Oxford 328.2002.

26. Schindelin, J.; Arganda-Carreras, I.; Frise, E.; Kaynig, V.; Longair, M.; Pietzsch, T.; Preibisch, S.; Rueden, C.; Saalfeld, S.; Schmid, B.; et al. Fiji: An open-source platform for biological-image analysis. Nat. Methods 2012.

27. Love, M.I.; Huber, W.; Anders, S. Moderated estimation of fold change and dispersion for RNA-seq data with DESeq2. Genome Biol.2014.

28. Fiorio Pla, A.; Kondratska, K.; Prevarskaya, N. STIM and ORAI proteins: crucial roles in hallmarks of cancer. Am. J. Physiol. Physiol.2016.

29. Prevarskaya, N.; Skryma, R.; Shuba, Y. Calcium in tumour metastasis: new roles for known actors. Nat. Rev. Cancer2011, 11, 609-618.

30. Yang, S.; Zhang, J.J.; Huang, X.Y. Orai1 and STIM1 Are Critical for Breast Tumor Cell Migration and Metastasis. Cancer Cel/2009.

31. Balthasar, S.; Bergelin, N.; Löf, C.; Vainio, M.; Andersson, S.; Törnquist, K. Interactions between sphingosine-1-phosphate and vascular endothelial growth factor signalling in ML-1 follicular thyroid carcinoma cells. Endocr. Relat. Cancer2008, 15, 521-534.

32. Vashisht, A.; Trebak, M.; Motiani, R.K. STIM and orai proteins as novel targets for cancer therapy. A review in the theme: Cell and molecular processes in cancer metastasis. Am. J. Physiol. - Cell Physiol.2015.

33. Kalhori, V.; Törnquist, K. MMP2 and MMP9 participate in S1P-induced invasion of follicular ML-1 thyroid cancer cells. Mol. Cell. Endocrinol.2015, 404, 113-122.

34. Lazar, V.; Bidart, J.M.; Caillou, B.; Mahé, C.; Lacroix, L.; Filetti, S.; Schlumberger, M. Expression of the $\mathrm{Na}+/$ I- symporter gene in human thyroid tumors: A comparison study with other thyroid-specific genes. J. Clin. Endocrinol. Metab.1999. 
35. De Martimprey, H.; Bertrand, J.R.; Fusco, A.; Santoro, M.; Couvreur, P.; Vauthier, C.; Malvy, C. siRNA nanoformulation against the Ret/PTC1 junction oncogene is efficient in an in vivo model of papillary thyroid carcinoma. Nucleic Acids Res.2008.

36. Niemelä, E.; Desai, D.; Niemi, R.; Doroszko, M.; Özliseli, E.; Kemppainen, K.; Rahman, N.A.; Sahlgren, C.; Törnquist, K.; Eriksson, J.E.; et al. Nanoparticles carrying fingolimod and methotrexate enables targeted induction of apoptosis and immobilization of invasive thyroid cancer. Eur. J. Pharm. Biopharm.2020.

37. Karacicek, B.; Erac, Y.; Tosun, M. Functional consequences of enhanced expression of STIM1 and Orai1 in Huh-7 hepatocellular carcinoma tumor-initiating cells. BMC Cancer2019.

38. Hanahan, D.; Weinberg, R.A. The hallmarks of cancer. Cel/ 2000.

39. Hanahan, D.; Weinberg, R.A. Hallmarks of cancer: The next generation. Cell 2011, 144, 646-674.

40. Prevarskaya, N.; Skryma, R.; Shuba, Y. Ion channels and the hallmarks of cancer. Trends Mol. Med. 2010, 16, 107-121.

41. Prevarskaya, N.; Skryma, R.; Shuba, Y. Ion Channels in Cancer: Are Cancer Hallmarks Oncochannelopathies? Physiol. Rev.2018.

42. Schmidt, S.; Liu, G.; Liu, G.; Yang, W.; Honisch, S.; Pantelakos, S.; Stournaras, C.; Hönig, A.; Lang, F. Enhanced Orai1 and STIM1 expression as well as store operated Ca2+ entry in therapy resistant ovary carcinoma cells. Oncotarget2014.

43. Chen, Y.F.; Chiu, W.T.; Chen, Y.T.; Lin, P.Y.; Huang, H.J.; Chou, C.Y.; Chang, H.; Tang, M.J.; Shen, M.R. Calcium store sensor stromal-interaction molecule 1-dependent signaling plays an important role in cervical cancer growth, migration, and angiogenesis. Proc. Natl. Acad. Sci. U. S. A.2011.

44. Sun, J.; Lu, F.; He, H.; Shen, J.; Messina, J.; Mathew, R.; Wang, D.; Sarnaik, A.A.; Chang, W.-C.; Kim, M.; et al. STIM1- and Orai1-mediated $\mathrm{Ca}(2+)$ oscillation orchestrates invadopodium formation and melanoma invasion. J. Cell Biol.2014.

45. Wang, J.Y.; Sun, J.; Huang, M.Y.; Wang, Y.S.; Hou, M.F.; Sun, Y.; He, H.; Krishna, N.; Chiu, S.J.; Lin, S.; et al. STIM1 overexpression promotes colorectal cancer progression, cell motility and COX-2 expression. Oncogene2015.

46. Zhao, S.; Huang, J.; Ye, J. A fresh look at zebrafish from the perspective of cancer research. J. Exp. Clin. Cancer Res. 2015.

47. Hason, M.; Bartůnĕk, P. Zebrafish models of cancer-new insights on modeling human cancer in a non-mammalian vertebrate. Genes (Basel). 2019.

48. Pyne, N.J.; El Buri, A.; Adams, D.R.; Pyne, S. Sphingosine 1-phosphate and cancer. Adv. Biol. Regul. 2018.

49. Bergelin, N.; Löf, C.; Balthasar, S.; Kalhori, V.; Törnquist, K. S1P1 and VEGFR-2 form a signaling complex with extracellularly regulated kinase $1 / 2$ and protein kinase $\mathrm{C}$-a regulating $\mathrm{ML}-1$ thyroid carcinoma cell migration. Endocrinology2010, 151, 2994-3005. 
50. Kalhori, V.; Kemppainen, K.; Asghar, M.Y.; Bergelin, N.; Jaakkola, P.; Törnquist, K. Sphingosine-1Phosphate as a Regulator of Hypoxia-Induced Factor-1a in Thyroid Follicular Carcinoma Cells. PLoS One2013, 8.

51. Al-Jundi, M.; Thakur, S.; Gubbi, S.; Klubo-Gwiezdzinska, J. Novel targeted therapies for metastatic thyroid cancer-a comprehensive review. Cancers (Basel).2020, 12, 1-37.

52. Kondratska, K.; Kondratskyi, A.; Yassine, M.; Lemonnier, L.; Lepage, G.; Morabito, A.; Skryma, R.; Prevarskaya, N. Orai1 and STIM1 mediate SOCE and contribute to apoptotic resistance of pancreatic adenocarcinoma. Biochim. Biophys. Acta - Mol. Cell Res.2014.

53. Rosenholm, J.M.; Peuhu, E.; Bate-Eya, L.T.; Eriksson, J.E.; Sahlgren, C.; Lindén, M. Cancer-cell-specific induction of apoptosis using mesoporous silica nanoparticles as drug-delivery vectors. Sma//2010.

54. De Martimprey, H.; Bertrand, J.R.; Malvy, C.; Couvreur, P.; Vauthier, C. New core-shell nanoparticules for the intravenous delivery of sirna to experimental thyroid papillary carcinoma. Pharm. Res.2010.

55. Zhou, Y.; Gu, P.; Li, J.; Li, F.; Zhu, J.; Gao, P.; Zang, Y.; Wang, Y.; Shan, Y.; Yang, D. Suppression of STIM1 inhibits the migration and invasion of human prostate cancer cells and is associated with $\mathrm{PI} 3 \mathrm{~K} / \mathrm{Akt}$ signaling inactivation. Oncol. Rep.2017.

56. Aashiq, M.; Silverman, D.A.; Na'ara, S.; Takahashi, H.; Amit, M. Radioiodine-refractory thyroid cancer: Molecular basis of redifferentiation therapies, management, and novel therapies. Cancers (Basel). 2019.

\section{Figures}



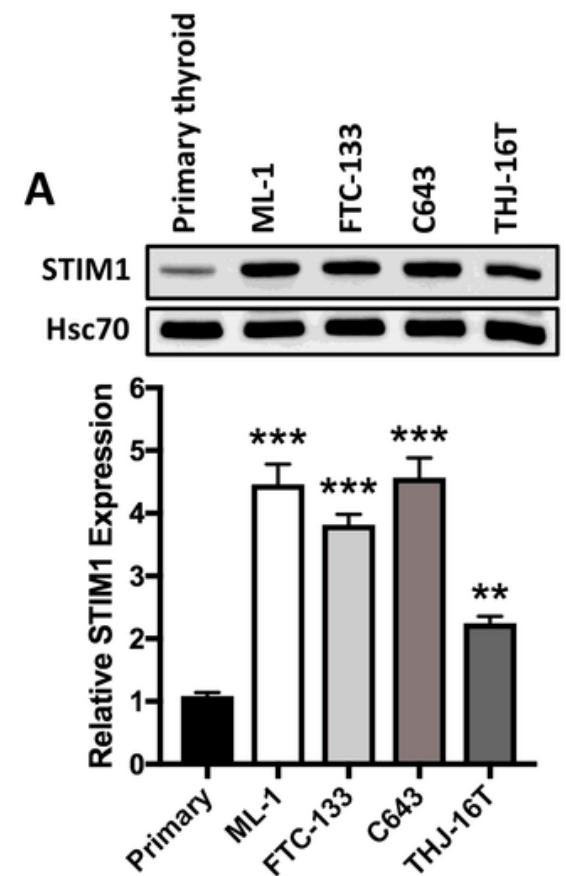

B

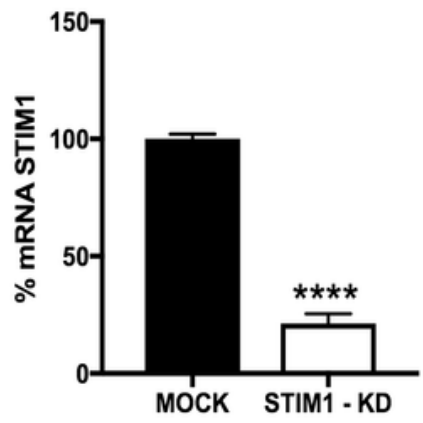

D

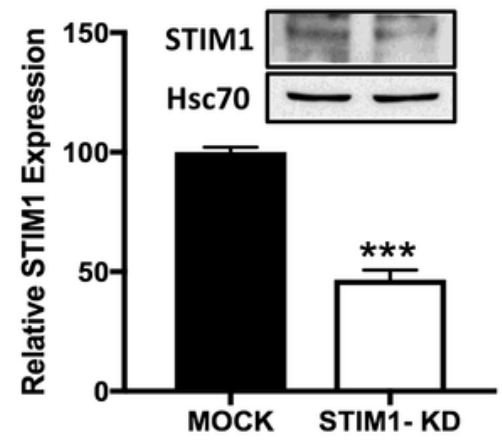

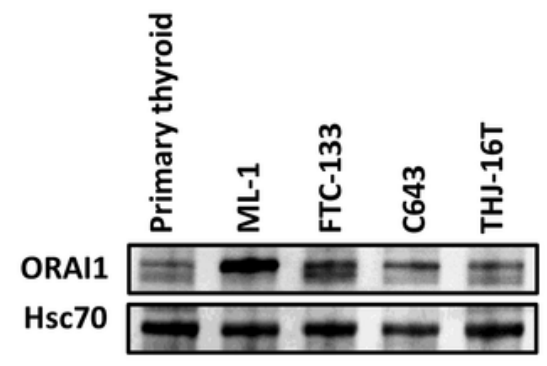
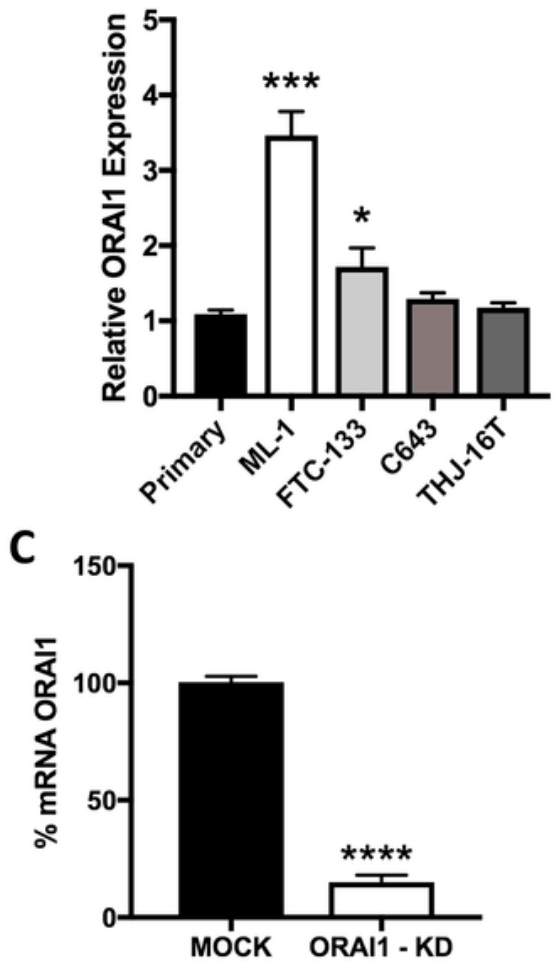

$\mathbf{E}$

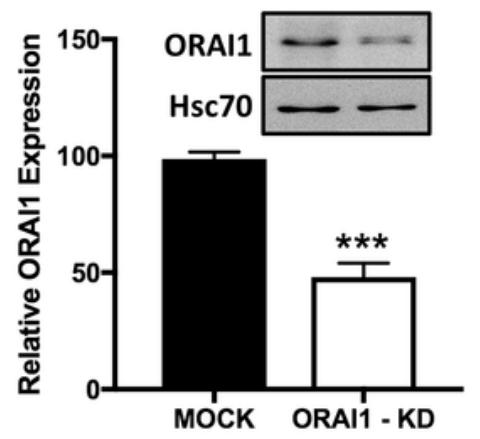

Figure 1

Expression of STIM1 and ORAI1 proteins and generation of stable knock-down cells. A, Expression profile of STIM1 and ORAl1 proteins in primary thyroid and aggressive thyroid cancer cell lines by western blotting. The bars show the mean \pm SEM $(n=3, *, P<0.05$, **, $P<0.01$; ***, $P<0.001)$. B-E, expression of STIM1 or ORAl1 in shRNA expressing (MOCK) and shRNA STIM1 expressing (STIM1-KD) or shRNA ORAI1 
expressing (ORAI1-KD) cells on mRNA and protein levels by RT-PCR and Western blotting, respectively. The bars show the mean \pm S.E. $(n=3)$. ***, $p<0.001$.
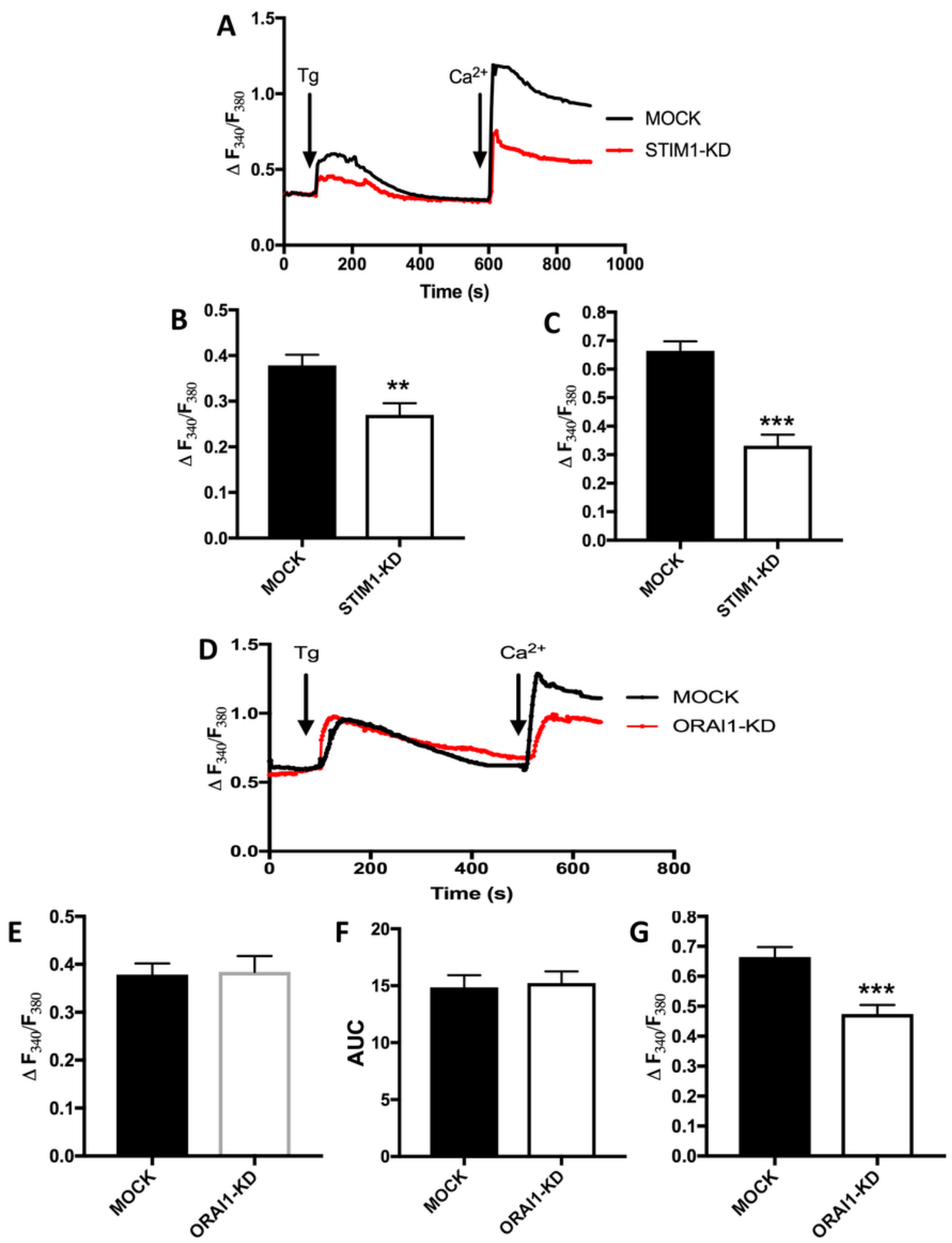

Figure 2

A, Representative traces showing Thapsigargin-evoked ( $\mathrm{Tg}$, final concentration, $1 \mu \mathrm{M})$ changes in $[\mathrm{Ca} 2+] \mathrm{i}$ in MOCK and STIM1-KD cells in calcium-free buffer and the effect of re-addition of $1 \mathrm{mM}$ calcium (final concentration). B, Bar diagram showing the magnitude of the Tg-evoked peak in [Ca2+]i. The bars show 
the mean \pm S.E. of at least 40 cells. ${ }^{* *}, p<0.01$. C, Bar diagram showing the magnitude of the change in [Ca2+]i after re-addition of calcium (final concentration, $1 \mathrm{mM}$ ) to Tg-treated cells. The bars show the mean \pm S.E. of at least 40 cells. $* \star *, p<0.001$. D, Representative traces showing Tg-evoked (final concentration, $1 \mu \mathrm{M}$ ) changes in [Ca2+]i in MOCK and ORAI1-KD cells in calcium-free buffer and the effect of readdition of $1 \mathrm{mM}$ calcium. E, Bar diagram showing the magnitude of the Tg-evoked peak in $[\mathrm{Ca} 2+]$ i. The bars show the mean \pm S.E. of at least 40 cells. F, Bar diagram showing the area under the $\mathrm{Tg}$ curve (time, $60-480 \mathrm{~s}$ ). The bars show the mean \pm S.E. of at least 40 cells. $G$, Bar diagram showing the magnitude of the change in [Ca2+]i after readdition of calcium (final concentration, $1 \mathrm{mM}$ ) to Tg-treated cells. The bars show the mean \pm S.E. of at least 40 cells. ${ }^{\star \star \star}, p<0.001$. 


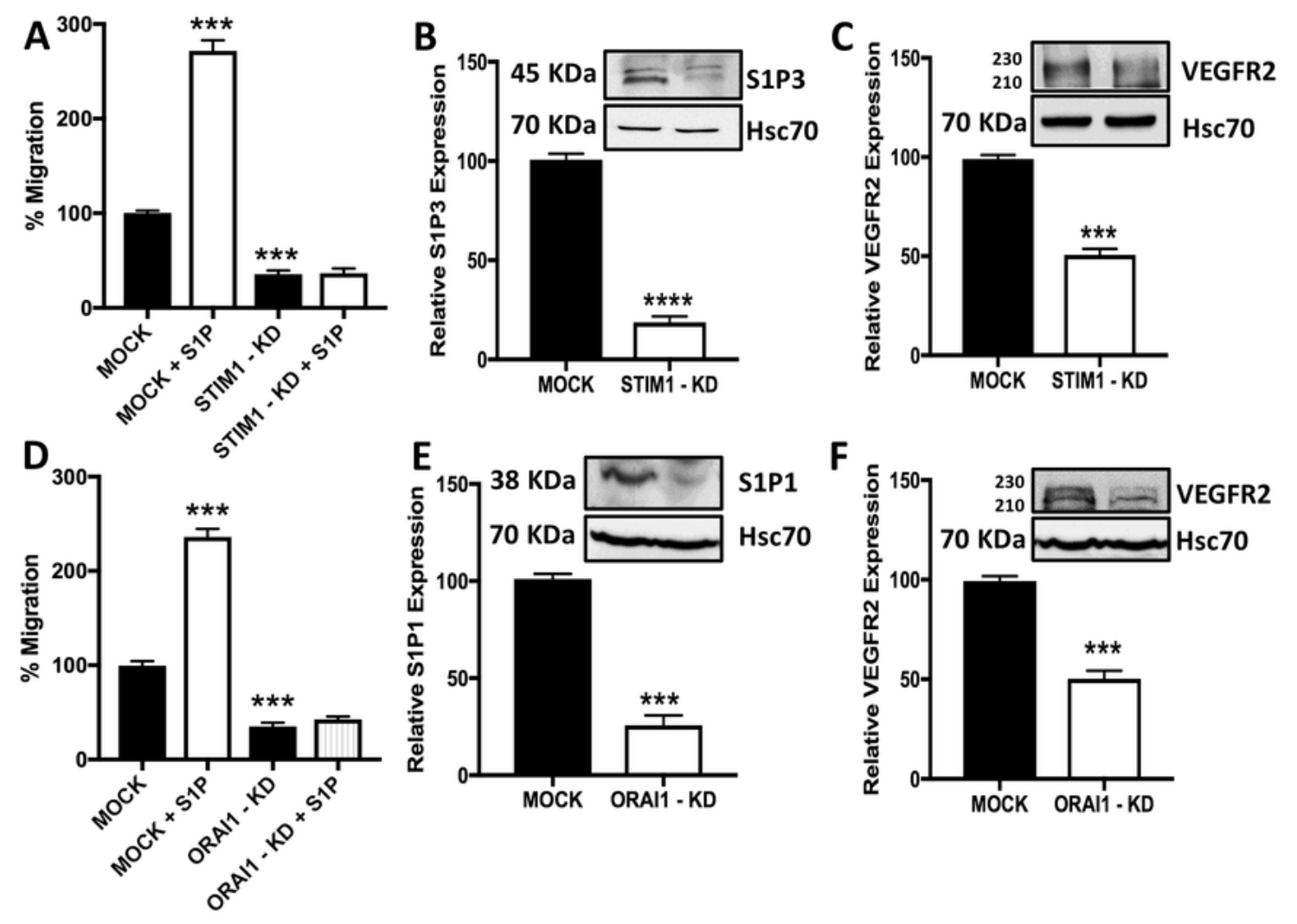

Figure 3

STIM1 or ORAI1 knock-down attenuates invasion and receptor expression in thyroid cancer ML-1 cells. A and D, STIM1 or ORAI1 knock-down both decreased the basal and abolished the S1P-evoked (final concentration, $100 \mathrm{~nm})$ invasion and migration in ML-1 cells. The bars show the mean \pm S.E. $(n=4)$. ***, $\mathrm{p}<0.001$. B, STIM1 knock-down decreased the relative expression of S1P3. A representative Western blot is shown. The bar diagram shows the decreased expression of S1P3 in STIM1-KD cells compared with 
MOCK cells (mean \pm S.E., $n=3$ ) ${ }^{\star \star \star}, p<0.001$. C, STIM1 knock-down decreased the relative expression of VEGFR2. A representative Western blot is shown. The bar diagram shows the decreased expression of VEGFR2 in STIM1-KD cells compared with MOCK cells (mean \pm S.E., $n=3$ ). ${ }^{\star * *}, p<0.001$. E, ORAl1 knockdown decreased the relative expression of S1P1. A representative Western blot is shown. The bar diagram shows the decreased expression of S1P1 in ORAl1-KD cells compared with MOCK cells (mean \pm S.E., $n=$ 3). $* \star *, p<0.001$. F, ORAl1 knock-down decreased the relative expression of VEGFR2. A representative Western blot is shown. The bar diagram shows the decreased expression of VEGFR2 in STIM1-KD cells compared with MOCK cells (mean \pm S.E., $n=3$ ). ***, $p<0.001$. 

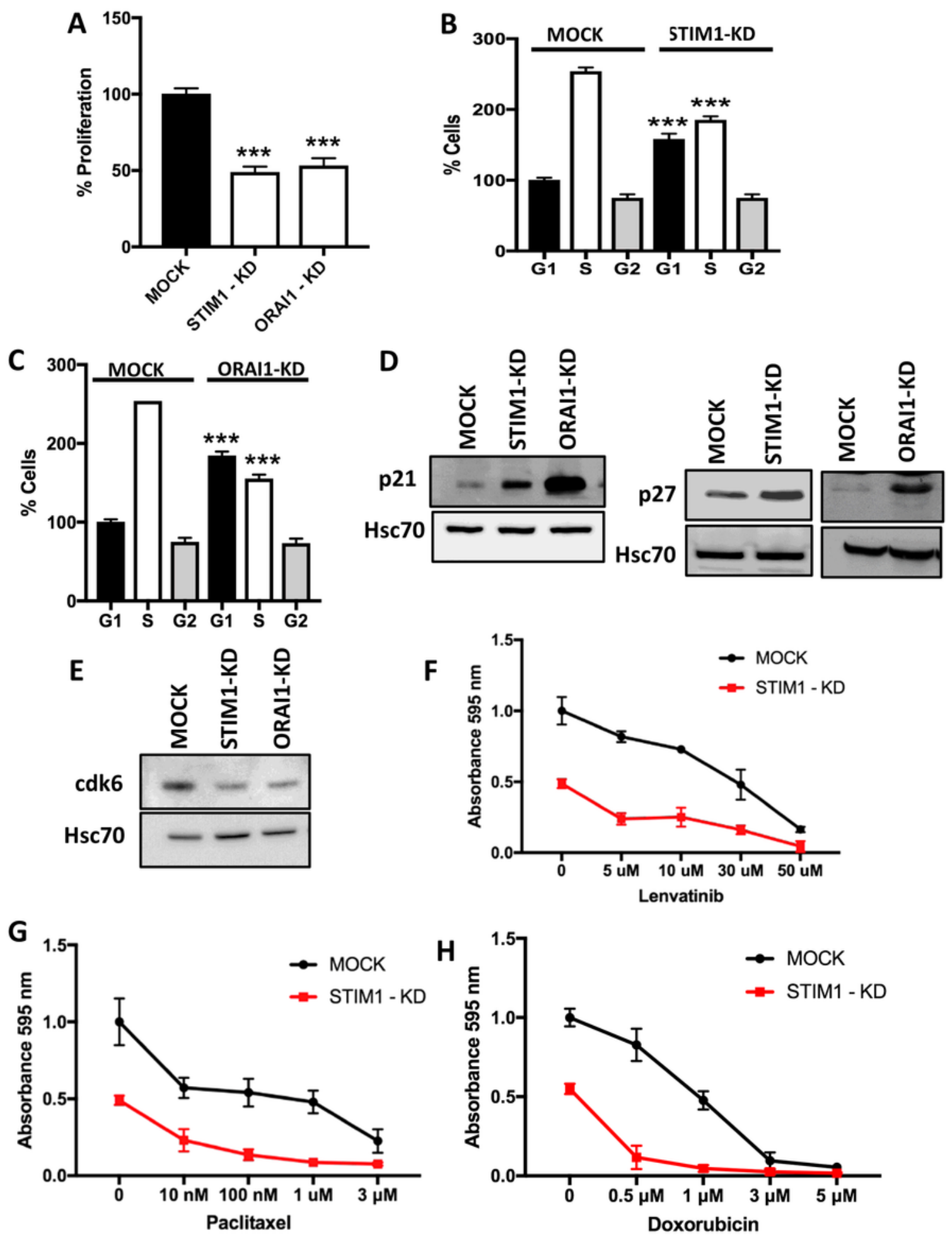

Figure 4

STIM1 and ORAI1 regulate proliferation of thyroid cancer ML-1 cells. A, STIM1 and ORAI1 knock-down decreased proliferation after $48 \mathrm{~h}$. The bar diagram shows the mean $\pm S$.E. $(n=3)$. $* \star *, p<0.001$. B and C, STIM1 and ORAl1 knock-down decreased proliferation by prolonging the G1 phase and decreasing the $S$ phases of the cell cycle. ${ }^{* \star}, \mathrm{p}<0.001$. ${ }^{*}$, statistically significant differences between the $\mathrm{G} 1$ and $\mathrm{S}$ phases of cell cycle, respectively, in STIM1-KD and ORAI1-KD cells compared with the MOCK cells. D, 
Representative Western blots showing the upregulation of p21 and p27 in both STIM1 and ORAI1-KD cells. E, Representative Western blot showing the downregulation of cyclin-dependent kinase 6 (cdk6) in both STIM1 and ORAI1-KD cells. F-H, Cells were treated with indicated concentrations of Lenvatinib, Paclitaxel or Doxorubicin and the proliferation was assessed by MTT proliferation assays $(24 \mathrm{~h}$. The effects of tested drugs were significant $(p<0.001)$ in STIM1-KD compared with MOCK cells. Each data point represents the mean \pm S.E. of at least three independent experiments.
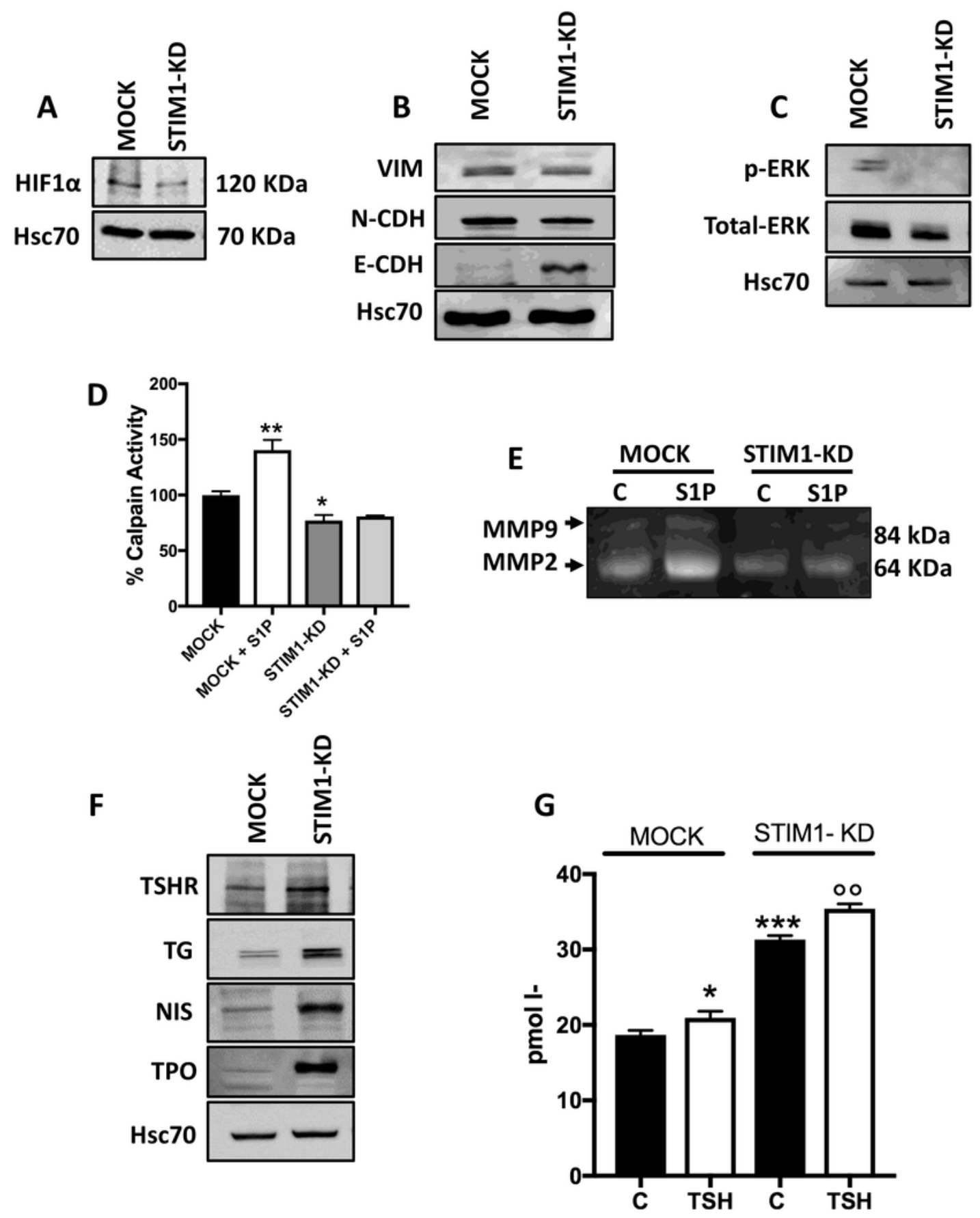

Figure 5 
STIM1 knock-down decreased the expression of HIF-1a, calpain activity and the activity of MMP2 and MMP9 and in ML-1 cells. A, the expression of HIF-1a was significantly decreased in STIM1-KD cells compared with MOCK cells. A representative Western blot is shown. B, the expression of EMT marker proteins in STIM1-KD cells compared to the MOCK ML-1 cells. The representative Western blots are shown. C, the expression of total-ERK and phospho-ERK in STIM1-KD cells compared with MOCK ML-1 cells. The representative Western blots are shown. D, STIM1 knock-down decreased the basal and abolished the S1P-evoked increase in calpain activity compared to MOCK ML-1 cells. $(\mathrm{N}=4)$, * denotes comparison with MOCK. ${ }^{*} p<0.05$, $* * p<0.01$. E, STIM1 knock-down increased the expression of thyroidspecific proteins. The representative Western blots of at least three independent experiments are shown. F, STIM1 knock-down enhanced the basal and TSH-evoked iodine-uptake compared to in the MOCK cells. *, denotes comparison with MOCK cells; o, denotes comparison with control STIM1-KD cells. $(n=4) * p<$ $0.05, * * p<0.01$, o0 $p<0.01$. 

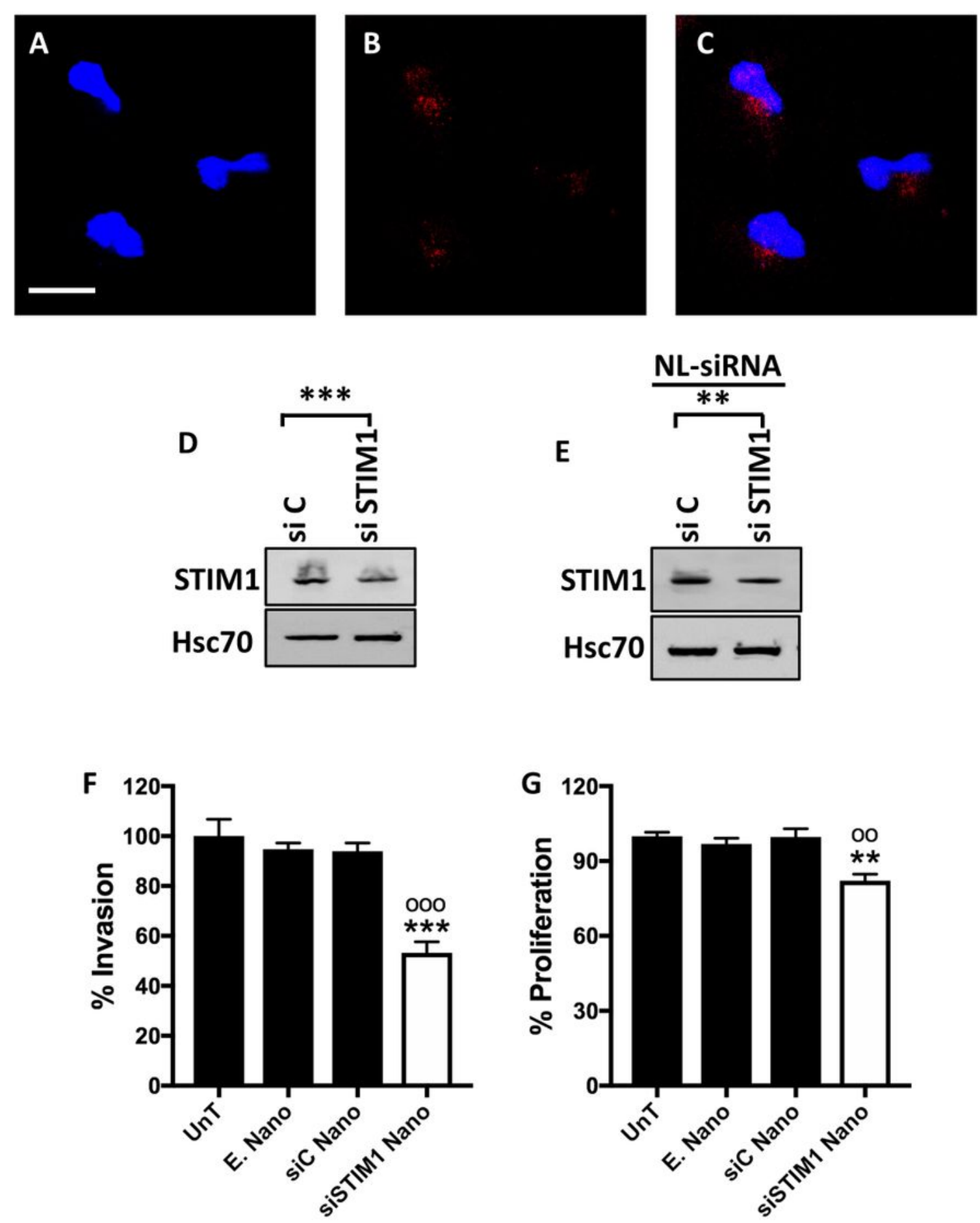

Figure 6

Functional efficacy of MPDA-DMEA nanoparticles in ML-1 thyroid cancer cells. A-C, Confocal images of ML-1 cells with control siRNA-Alexa Fluor 555 loaded nanoparticles. A, (DAPI), B, (siRNA-Alexa Fluor 555), and C, (Merge: DAPI + siRNA-Alexa Fluor 555). The scale bar is $20 \mu \mathrm{m}$. The images shown are representative of at least three separate experiments. D, Representative western blot showing the efficacy of siSTIM1 in ML-1 cells. E, Representative western blot showing the efficacy of siSTIM1-loaded MPDA- 
DMEA nanoparticles in ML-1 cells. NL-siRNA denotes nanoparticles loaded siRNA. F, G, siSTIM1-loaded nanoparticles decreased the migration and proliferation of ML-1 cells. UnT, un-treated; E.Nano, empty MPDA-DMEA nanoparticles, siC Nano, control non-target siRNA loaded nanoparticles, siSTIM1 Nano, siSTIM1 loaded nanoparticles. *, ML-1 unT vs siSTIM1 Nano, o, siC Nano vs siSTIM1 Nano. The bars show the mean \pm S.E. $(n=6)$. ${ }^{\star *}, p<0.001 ; 000, p<0.001$.
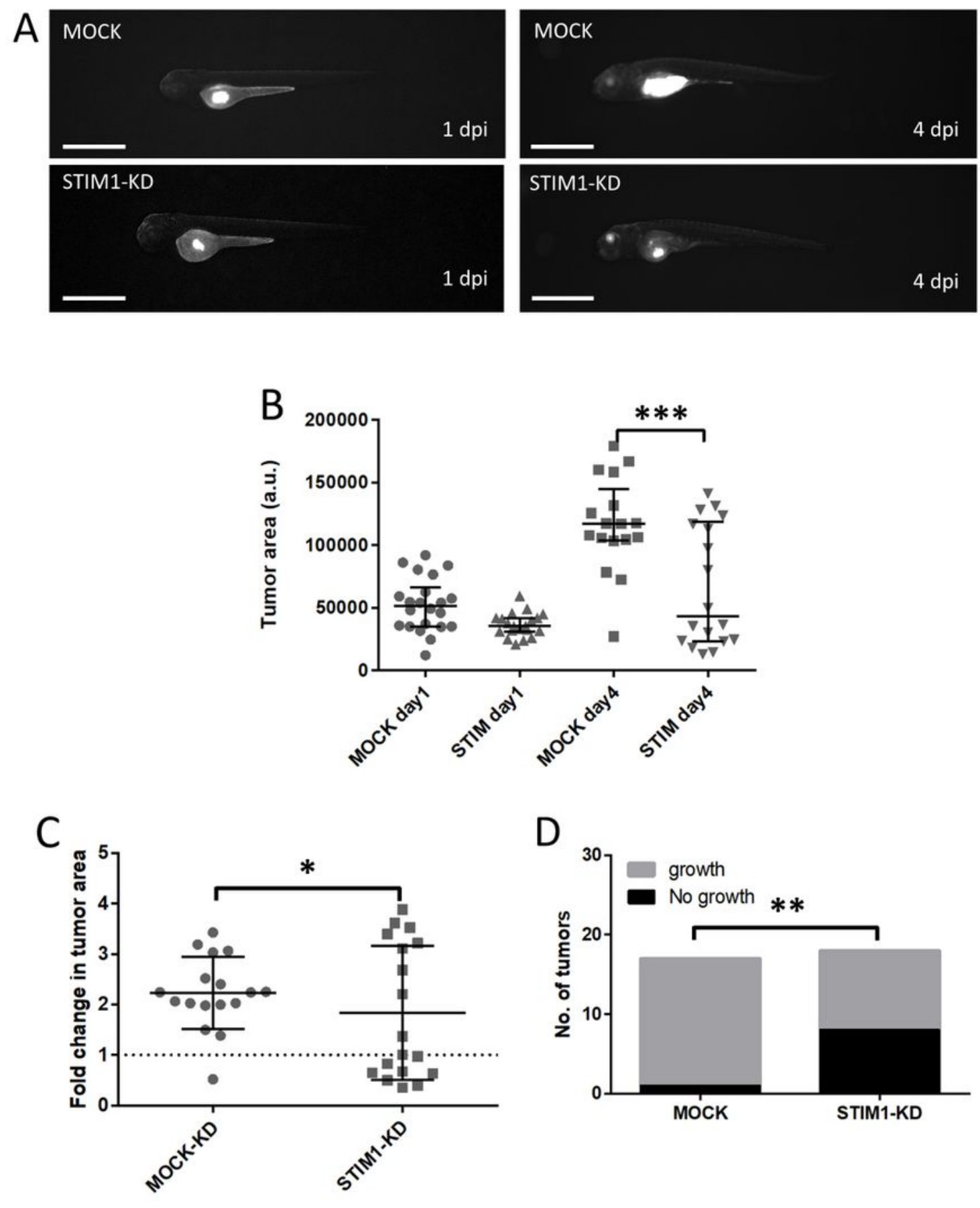

Figure 7 
Zebrafish xenograft experiment. A) Representative images of one and four day (s) post injection (dpi) with MOCK and STIM1-KD cells and following the tumors growth. The scale bar is $1 \mathrm{~mm}$. B, C) Quantitation of tumor size and fold change area. D) Quantitation of mortality and survival of embryos E) Quantitation of tumor growth. ${ }^{*} p<0.05 ; * * p<0.01 ; * * * p<0.001$.

A

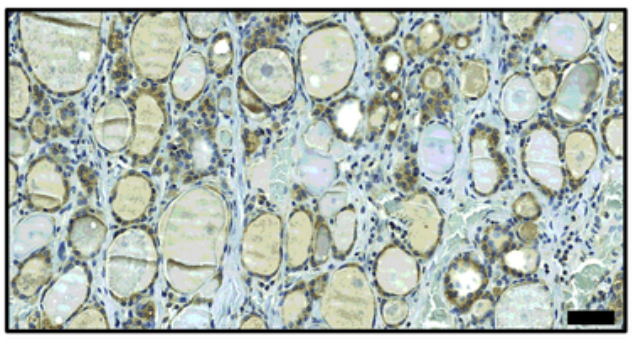

C

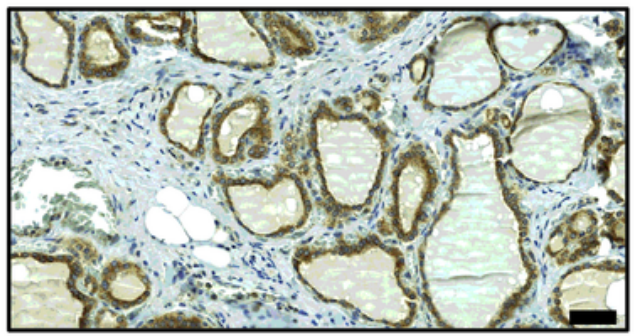

E

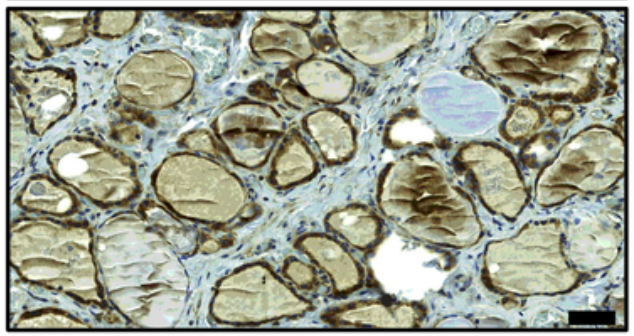

G

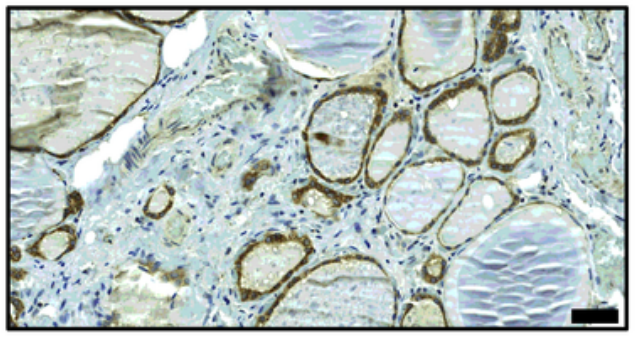

B

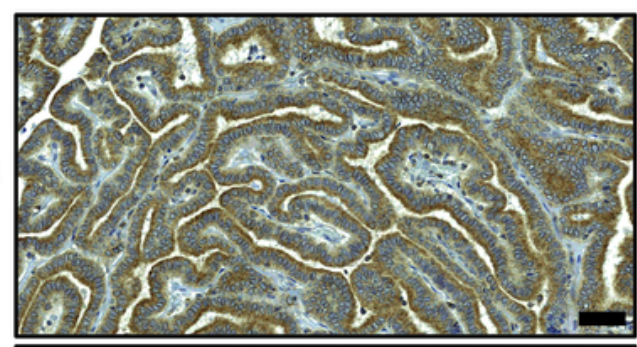

D

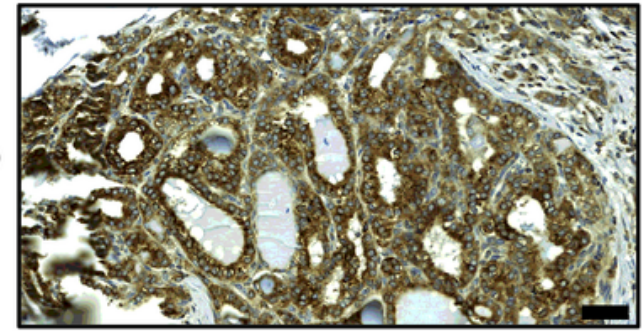

F

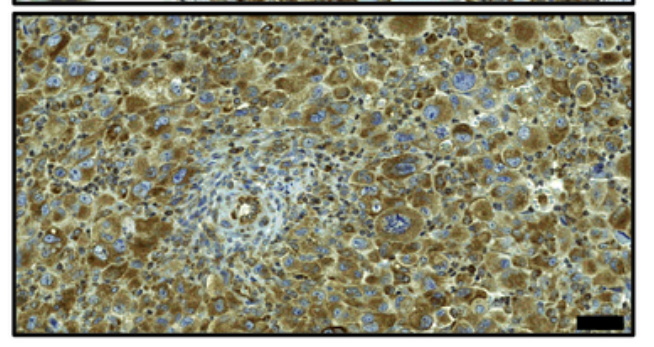

H
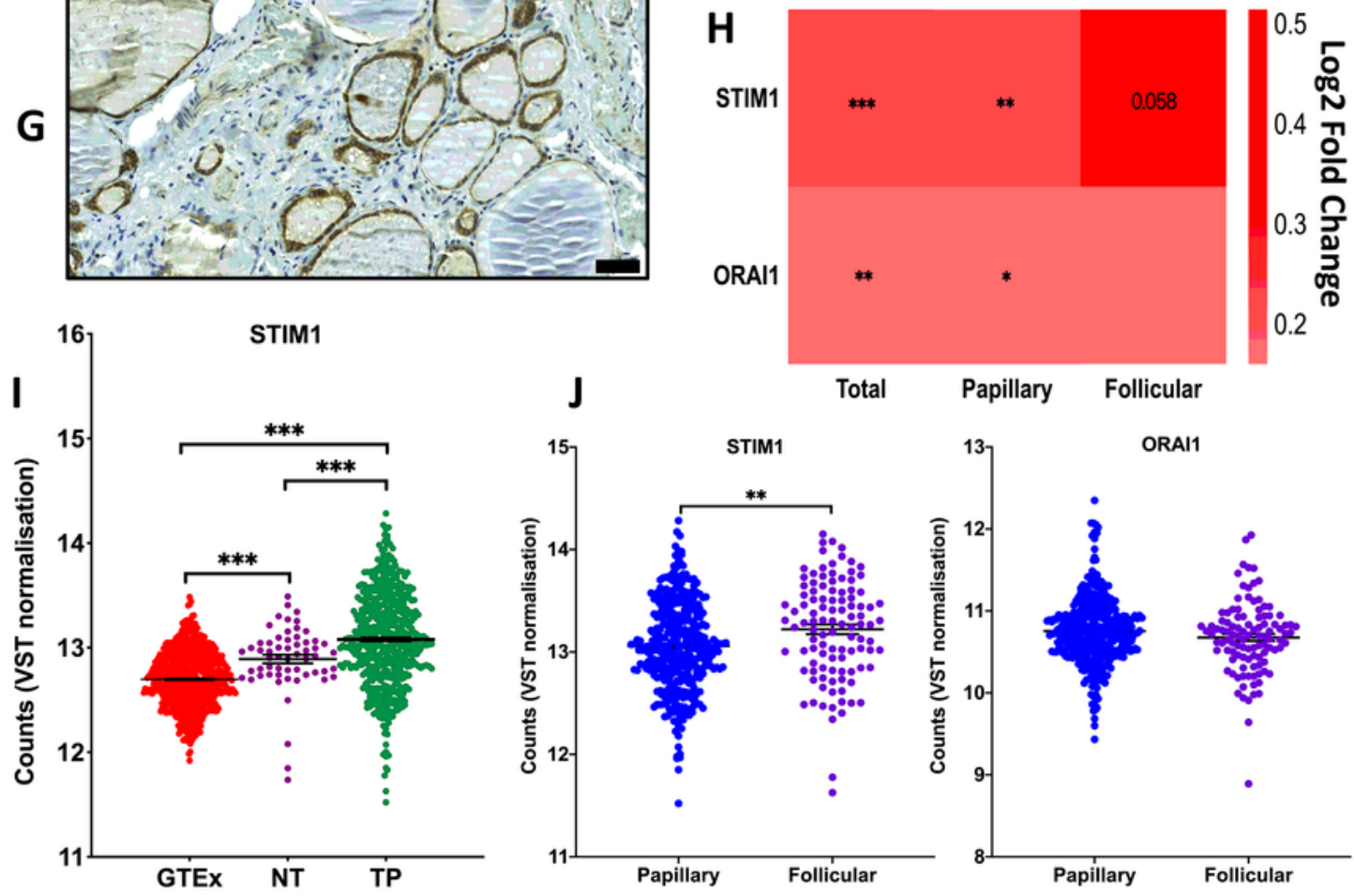

Papillary Follicular

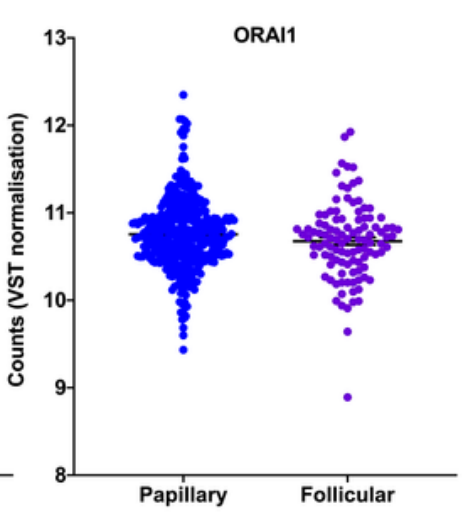

Figure 8 
STIM1-protein expression in human normal and thyroid cancer tissues. A-B. Representative normal and anaplastic thyroid cancer. C-D. Representative normal and papillary thyroid cancer tissue. E-F.

Representative normal and follicular thyroid cancer tissue. The representative control and cancer tissue sections of each cancer type are from the same patient. G. Representative normal thyroid tissue from a healthy subject. Scale bar $50 \mu \mathrm{M}$. The final images were acquired at 20X magnification using case viewer software (3DHITECH Ltd, Germany). H: Heatmap to visualize differential expression of STIM1 and ORAI1 between thyroid tumors and their adjacent normal samples. The color intensity represents log2 Fold Changes. Significances have been corrected by Benjamini-Hochberg method. I: STIM1 expression comparison between thyroid cancer tissues, their para-cancerous tissues, and normal controls. Data were presented as mean \pm SE. One-way ANOVA test was used to assess statistical significance. J: STIM1 and ORAI1 expression comparison between papillary thyroid cancer tissues and follicular thyroid cancer tissues. Data were presented as mean $\pm \mathrm{SE}$. Unpaired t-test was used to assess statistical significance. ${ }^{*} \mathrm{p}$ $<0.05 ; * \star p<0.01 ; * \star \star p<0.001$.

\section{Supplementary Files}

This is a list of supplementary files associated with this preprint. Click to download.

- SupplementaryFigure.1.tif

- SupplementaryTable1.tif 